\title{
Balancing functions of annexin A6 maintain equilibrium between hypertrophy and apoptosis in cardiomyocytes
}

\author{
P Banerjee ${ }^{1}$, V Chander $^{1}$ and A Bandyopadhyay ${ }^{* 1}$
}

Pathological cardiac hypertrophy is a major risk factor associated with heart failure, a state concomitant with increased cell death. However, the mechanism governing progression of hypertrophy to apoptosis at the single-cell level remains elusive. Here, we demonstrate annexin A6 (Anxa6), a calcium $\left(\mathrm{Ca}^{2+}\right.$-dependent phospholipid-binding protein critically regulates the transition of chronic hypertrophied cardiomyocytes to apoptosis. Treatment of the H9c2(2-1) cardiomyocytes with hypertrophic agonists upregulates and relocalizes Anxa6 with increased cytosolic punctate appearance. Live cell imaging revealed that chronic exposure to hypertrophic agonists such as phenylephrine (PE) compromises the mitochondrial membrane potential $\left(\Delta \Psi_{m}\right)$ and morphological dynamics. Such chronic hypertrophic induction also activated the caspases 9 and 3 and induced cleavage of the poly-(ADP-ribose) polymerase 1 (Parp1), which are the typical downstream events in the mitochondrial pathways of apoptosis. An increased rate of apoptosis was evident in the hypertrophied cardiomyocytes after 48-72 $\mathrm{h}$ of treatment with the hypertrophic agonists. Anxa6 was progressively associated with the mitochondrial fraction under chronic hypertrophic stimulation, and Anxa6 knockdown severely abrogated mitochondrial network and dynamics. Ectopically expressed Anxa6 protected the mitochondrial morphology and dynamics under PE treatment, and also increased the cellular susceptibility to apoptosis. Biochemical analysis showed that Anxa6 interacts with Parp1 and its $89 \mathrm{kDa}$ cleaved product in $\mathrm{C} \mathrm{Ca}^{2+}$-dependent manner through the $\mathrm{N}$-terminal residues (1-28). Furthermore, expression of Anxa6 ${ }^{\mathrm{S} 3 \mathrm{E}}$, a mutant dominant negative with respect to Parp1 binding, served as an enhancer of mitochondrial dynamics, even under chronic PE treatment. Chemical inhibition of Parp1 activity released the cellular vulnerability to apoptosis in Anxa6-expressing stable cell lines, thereby shifting the equilibrium away from cell death. Taken together, the present study depicts a dual regulatory function of Anxa6 that is crucial for balancing hypertrophy with apoptosis in cardiomyocytes.

Cell Death and Disease (2015) 6, e1873; doi:10.1038/cddis.2015.231; published online 3 September 2015

Complex machineries govern the life and death decisions in mammalian cells through a dynamic equilibrium, which is essential for physiological homeostasis. ${ }^{1}$ Such equilibrium is critical for cardiac myocytes because of their terminally differentiated states and low proliferative capacities. Stress response in cardiomyocytes often involves a switch between survival and cell death pathways. ${ }^{2-4}$ Cardiomyocyte hypertrophy is an adaptive response to stress, which may turn maladaptive and fatal, ${ }^{5}$ as evident in cardiovascular disorders that leads to heart failure. ${ }^{6}$ Hypertrophied phenotypes are also associated with a balance between cell growth and programmed cell death. ${ }^{7}$ These processes are aided by several patrolling proteins, which sense and operate to ameliorate the anomalies. ${ }^{8,9}$ Understanding the dynamics of such signaling events is vital for the development of novel therapeutic strategies.

Anxa6 belongs to the annexin family of calcium $\left(\mathrm{Ca}^{2+}\right) /$ phospholipid-binding proteins. ${ }^{10} \mathrm{~A}$ major cardiac annexin, ${ }^{11}$ Anxa6 has diverse functions ranging from handling intracellular
$\mathrm{Ca}^{2+}$ signaling, cholesterol transport, ${ }^{12}$ Ras inactivation ${ }^{13}$ and vesicular traffic. ${ }^{14}$ Anxa6 mostly functions as an intracellular scaffold. ${ }^{15}$ Although mice with targeted depletion of the Anxa6 gene remain viable, ${ }^{16}$ functional redundancies within the annexin family have been proposed to compensate for the loss of Anxa6 function. ${ }^{17,18}$ A 10-fold overexpression of Anxa6 targeted to the heart developed cardiomyopathies in mice, whereas cardiomyocytes from Anxa6-knockout mice exhibited increased contractility and altered $\mathrm{Ca}^{2+}$ turnover. ${ }^{19,20}$ Such contradictory findings may indicate participation of Anxa6 in counterbalancing signaling mechanisms. Moreover, end-stage heart failures have been reported to be associated with downregulation of Anxa6, and, in general, Anxa6 has compensatory roles in chronic pathological conditions. ${ }^{20-22}$ However, the function of differential Anxa6 expression or dynamics in chronic cardiomyocyte hypertrophy is poorly understood.

We have reported the interactions of Anxa6 with the sarcomeric $\alpha$-actinin and its role in cardiomyocyte contractility. ${ }^{23}$

\footnotetext{
${ }^{1}$ Cell Biology and Physiology Division, Council of Scientific and Industrial Research - Indian Institute of Chemical Biology, 4, Raja SC Mullick Road, Kolkata 700 032, West Bengal, India

*Corresponding author: A Bandyopadhyay, Cell Biology and Physiology Division, Council of Scientific and Industrial Research - Indian Institute of Chemical Biology, 4, Raja SC Mullick Road, Kolkata 700 032, India. Tel: +91 0 9433516720; Fax: +91 33 24995791/844; E-mail: arunb@iicb.res.in or arunb0925@yahoo.com

Abbreviations: Ang II, angiotensin II; ANP, atrial natriuretic peptide; Anxa6, annexin A6; $\alpha$-SkA, $\alpha$-skeletal actin; BAPTA-AM, 1, 2-Bis (2-aminophenoxy) ethane- $N$, N, $N^{\prime}$, $N^{\prime}$-tetraacetic acid tetrakis (acetoxymethyl ester); $\mathrm{Ca}^{2+}$, calcium; Co-IP, co-immunoprecipitation; COX I, anti-OxPhos complex IV subunit I; COX IV, cytochrome $c$ oxidase subunit IV; $\Delta \Psi \mathrm{m}$, mitochondrial membrane potential; FL, full length; FRAP, fluorescence recovery after photobleaching; IB, immunoblot; IP, immunoprecipitation; Iso, isoproterenol; LMB, leptomycin B; NRVM, neonatal rat ventricular myocyte; Parp1, poly-(ADP-ribose) polymerase 1; PE, phenylephrine; PI, propidium iodide; PInh, Parp1 inhibitor; pro-ANP, propeptide of atrial natriuretic peptide/intracellular precursor of atrial natriuretic peptide; Scramb, scrambled control; TMRM, tetramethylrhodamine; WCL, whole-cell lysate

Received 19.4.2015; revised 09.7.2015; accepted 10.7.2015; Edited by A Stephanou
} 
Recently, we have characterized a role of Anxa6 in the antihypertrophic signaling via the regulation of atrial natriuretic peptide (ANP) secretion. ${ }^{24}$ The mechanistic spectrum of Anxa6 in the earlier study was limited to a short-term (24 h) exposure of H9c2 cardiomyocytes to the a1-adrenergic receptor agonist phenylephrine (PE). The dynamics of Anxa6 within this small window yielded valuable insight into the spatiotemporal regulation of hypertrophic signaling. Here, we extended the study to understand the dynamics of Anxa6 under chronic hypertrophic conditions. The mechanodeficient $\mathrm{H} 9 \mathrm{c} 2(2-1)$ cardiomyocyte line has been instrumental in our study to rule out the contributions of Anxa6 towards contractility, ${ }^{23}$ owing to its multidimensional scaffold activity and functional compensations. ${ }^{17,18}$ The H9c2 cardiomyocytes have been extensively characterized and ARE an established animal origin-free model for studying signal-transduction pathways in cardiomyocytes, including hypertrophy. ${ }^{25,26}$

Adrenergic stimulation is crucial in compensatory and pathological cardiac hypertrophy, an early state that may proceed towards heart failure. ${ }^{27}$ Cardiac hypertrophy at advanced stages (chronic) is associated with mitochondrial dysfunction, which also contributes to cardiac decompensation. ${ }^{28}$ To explore the temporal events under chronic hypertrophy, we analyzed the effects of adrenergic induction on mitochondrial membrane potential $\left(\Delta \Psi_{\mathrm{m}}\right)$ and morphological dynamics, parameters that are directly correlated with mitochondrial dysfunction and programmed cell death. ${ }^{29-31}$ Anxa6 has been reported to be associated with mitochondria in some cell types. ${ }^{17,32,33}$ In the present study, we aim to understand the functions of Anxa6 under chronic hypertrophic conditions that may progress towards apoptosis.

\section{Results}

Chronic hypertrophy of $\mathrm{H} 9 \mathrm{c} 2$ cardiomyocytes is concomitant with altered expression and localization dynamics of Anxa6. H9c2 cardiomyocytes treated with PE were assessed for cellular hypertrophy by actin staining at the indicated time points (Figure 1). Compared with treatment with the hypertrophic agonists angiotensin II (Ang II) or isoproterenol (Iso), PE exhibited a linear response with time (Figure 2a), and was used in subsequent experiments. Increase in cell size was significant between 24 and $48 \mathrm{~h}$, which reached a plateau between 48 and $72 \mathrm{~h}$ (Figure 2a). Imaging of cells using atomic force microscope showed prominent increase in cellular height compared with control at $48 \mathrm{~h}$ (Supplementary Movies 1 and 2). Staining pattern for Anxa6 at 0 and $24 \mathrm{~h}$ time points confirmed our earlier results, ${ }^{24}$ depicting punctate cytosolic and membrane localization (Figure 1). The number of cells with Anxa6 puncta increased significantly with the duration of treatment (Figure $2 b$ ), parallel to an increase in the number and size of the punctate structures (Figures $2 b$ and $c$ ).

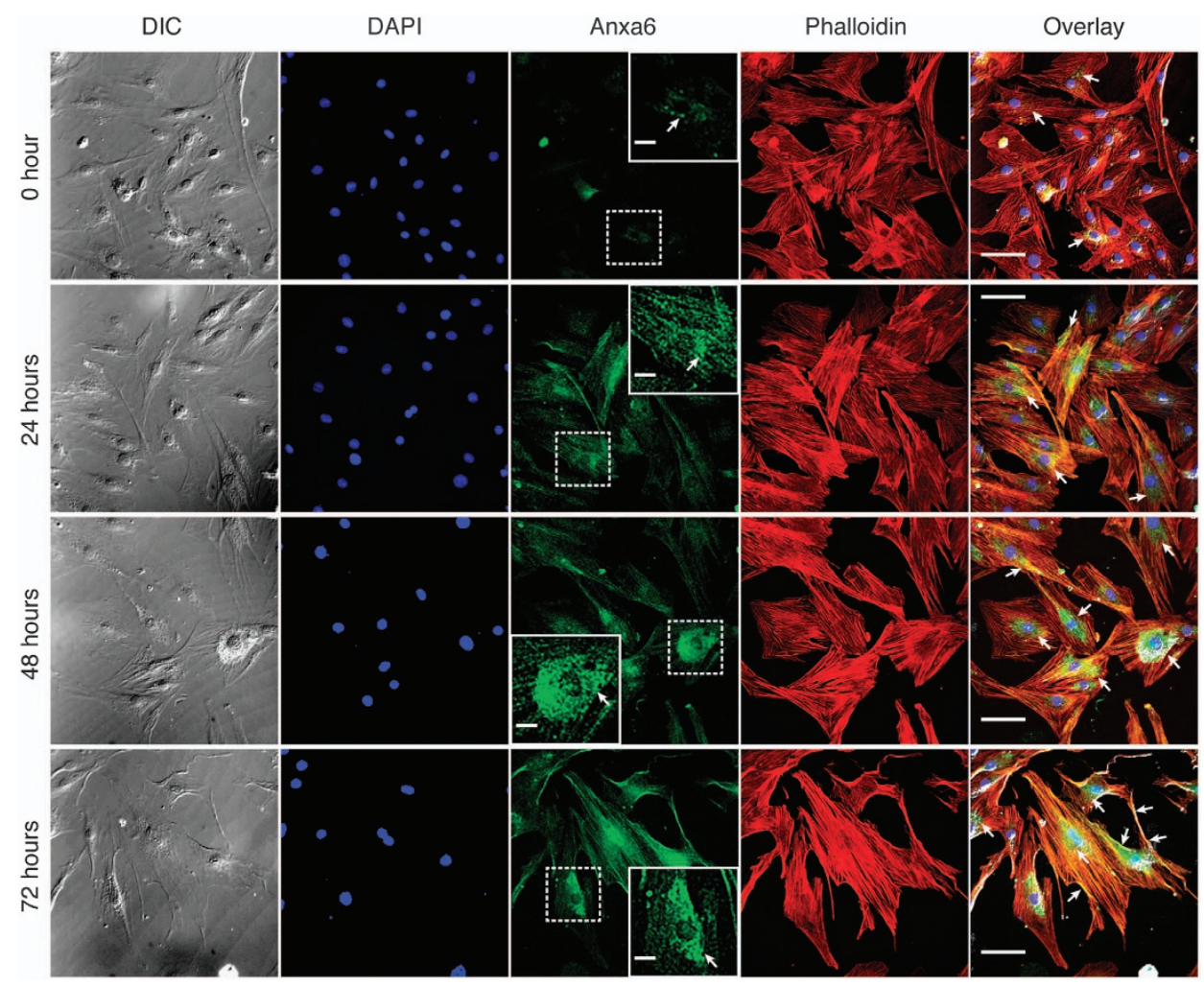

Figure 1 Relocalization dynamics of Anxa6 with chronic hypertrophic development. H9c2 cardiomyocytes were treated with $100 \mu \mathrm{M} P E$ for $72 \mathrm{~h}$ and were costained for Anxa6 and F-actin by ICC-IF (pseudocolored green) and Alexa Fluor 546 phalloidin (pseudocolored red), respectively, at indicated time points. Nucleus was counterstained with DAPI (4',6-diamidino-2-phenylindole; pseudocolored blue). Overlay represents maximum intensity projections of RGB channels merged with DIC. Arrowheads, distribution of punctate signals. Confocal images at $\times 600$ total magnification. Scale bar, $40 \mu \mathrm{m}$. Insets, punctate structures in the juxtanuclear compartment. Scale bar, $5 \mu \mathrm{m}$ 
Immunofluorescence as well as western blot analysis revealed that increased cell size was accompanied with elevated expression of Anxa6, as well as the hypertrophy markers pro-ANP (propeptide of ANP/intracellular precursor of ANP) and $a$-SkA ( $a$-skeletal muscle actin), which further confirmed augmented hypertrophic responses at 48 and $72 \mathrm{~h}$ (Figures 2d-f). Immunostaining for pro-ANP also revealed perinuclear accumulation of ANP secretory granules that significantly increased with the duration of the treatment (Figures $2 \mathrm{~g}$ and $\mathrm{h}$ ).

\section{Chronic hypertrophic induction abrogated mitochondrial} dynamics in $\mathrm{H9c2}$ cardiomyocytes. As the difference in cell size between 48 and $72 \mathrm{~h}$ was not significant (Figure 2a), we questioned whether the prohypertrophic signaling switches off during that period or culminates into other phenotypes. Hypertrophic signaling significantly overlaps with intrinsic/mitochondrial apoptotic pathways, without necessarily terminating into apoptosis. ${ }^{34}$ However, the transition from compensatory hypertrophy to heart failure is associated with increased cardiomyocyte death., ${ }^{2,35}$ This raises the possibility of a dynamic equilibrium between hypertrophy and apoptosis, which may act as a feedback loop for cellular homeostasis. We found that chronic stimulation of $\mathrm{H} 9 \mathrm{c} 2$ cardiomyocytes with the hypertrophic agonists $\mathrm{PE}$, Ang II or Iso compromised $\Delta \psi \mathrm{m}$, as revealed by a significant drop in tetramethylrhodamine (TMRM) fluorescence (Supplementary Figure 1A) as well as diminished $\mathrm{red} / \mathrm{green}$ ratio of the potential-dependent dye $\mathrm{JC}-1$, in the PE-treated cells (Figure $3 a$ and Supplementary Figure 1B). Immunostaining of COX I (anti-OxPhos complex IV subunit I) displayed reduced tubular mitochondria, with prominent a

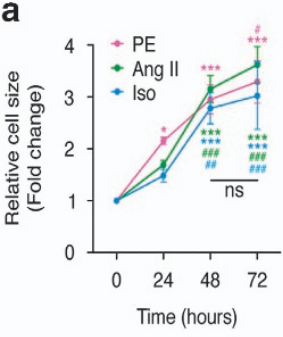

d

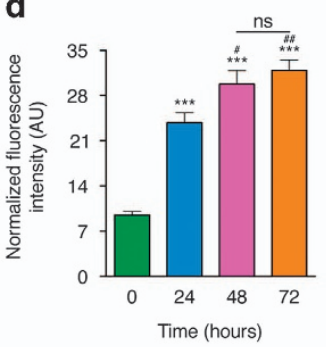

f

ㅁ 0 hour $\square 24$ hours

ㅁ 48 hours $\square 72$ hours ns

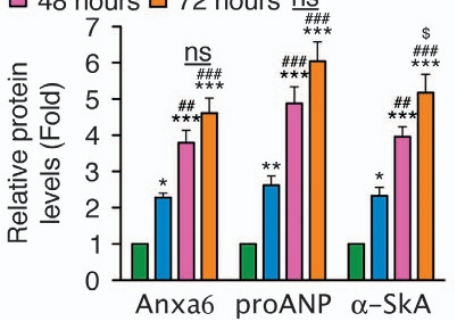

b

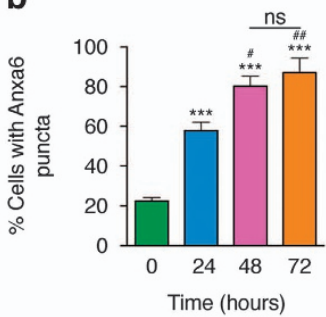

e

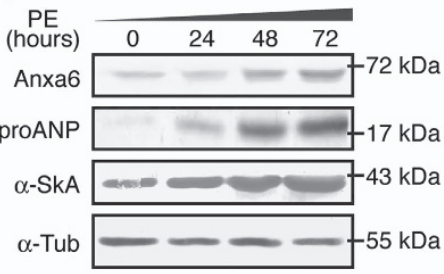

h

* \% Cellls with perinuclear ring

- Norm. Fl. Int. (AU)

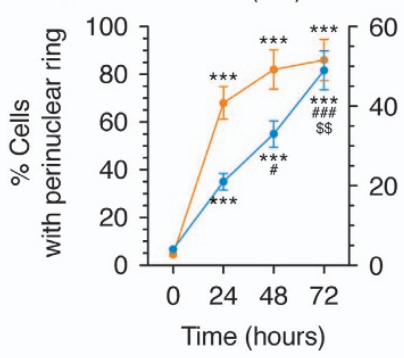

g
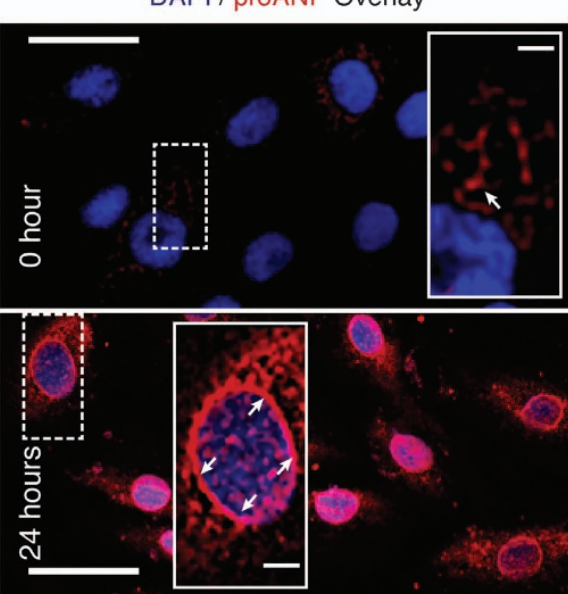

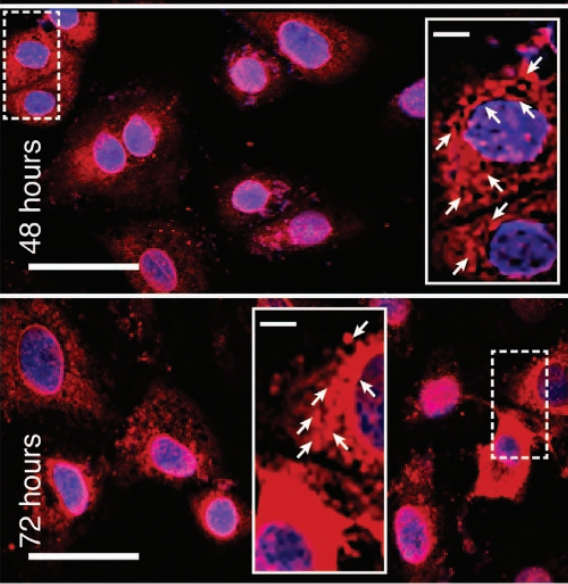

Figure 2 Quantitative assessment of Anxa6 dynamics in cardiomyocytes with chronic hypertrophied phenotypes. H9c2 cells treated with $100 \mu \mathrm{M}$ PE or $200 \mathrm{nM}$ Ang II or $10 \mu \mathrm{M}$ Iso for $72 \mathrm{~h}$ were analyzed for hypertrophy-associated transformations. (a) Quantification of cell size by staining with Alexa Fluor 546 phalloidin, as shown in Figure 1;

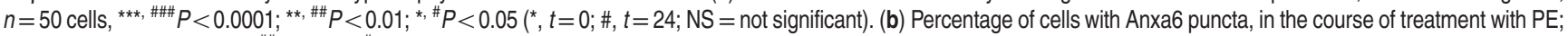
$n=10$ fields, ${ }^{* *} P<0.0001 ;{ }^{\# \#} P<0.01 ;{ }^{\#} P<0.05$ ( ${ }^{*}, t=0$; \#, $t=24$; NS $=$ not significant). (c) Number (left Yaxis) and diameter (right Yaxis) of Anxa6 punctate structures from Figure 1; $n=30$ cells; ${ }^{* * *}, \# \# \#<0.0001 ;{ }^{* *}, \# \#<0.01 ;{ }^{*} P<0.05$ ( ${ }^{*}, t=0$; \#, $\left.t=24\right)$. (d) Anxa6 expression analysis measured from immunofluorescent staining of cells shown in Figure 1; $n=30$ cells; ${ }^{* * *} P<0.0001 ;{ }^{\# \#} P<0.01 ; " \#<0.05$ ( ${ }^{*}, t=0$; \#, $t=24$; NS = not significant). (e) Immunoblot: protein levels of Anxa6, pro-ANP and $\alpha$-SkA. $\alpha$-Tubulin (Tub):

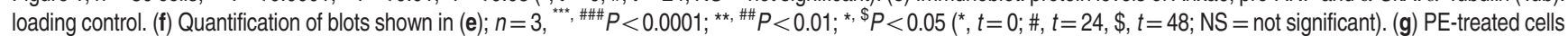
immunostained for pro-ANP. Nuclei counterstained with DAPI (4',6-diamidino-2-phenylindole). Total magnification $\times 630$. Scale bar, $40 \mu \mathrm{m}$. Insets, juxtanuclear pro-ANP clusters. Arrowheads, perinuclear signal. Scale bar, $3 \mu \mathrm{m}$. (h) Plot showing the percentage of cells expressing perinuclear pro-ANP ring (left $Y$ axis) and expression level of pro-ANP measured by normalized fluorescence intensity (right $Y$ axis) of red channel from $(\mathbf{g}) ; n=30$ cells; ${ }^{* * *}, \# \# \#<0.0001 ;{ }^{\$ \$} P<0.01 ;{ }^{\#} P<0.05$ ( ${ }^{*}, t=0$; \#, $t=24 ; \$, t=48$ ) 
a
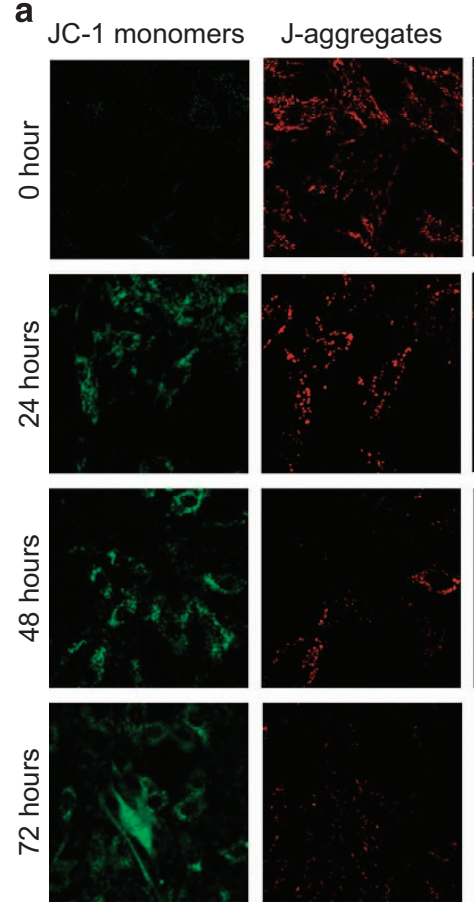

d

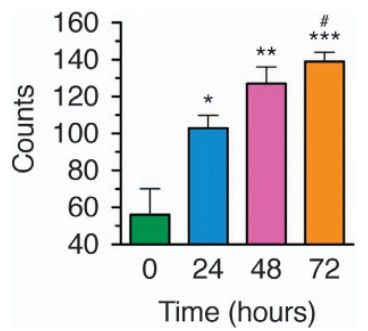

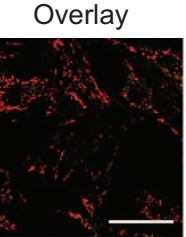
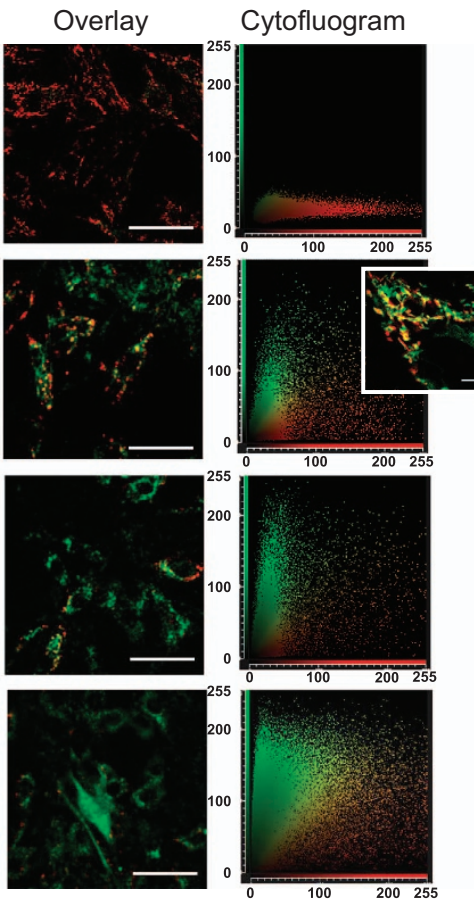

e

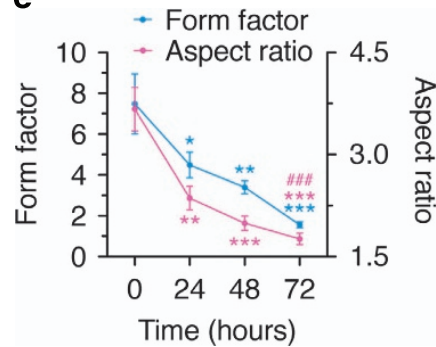

b

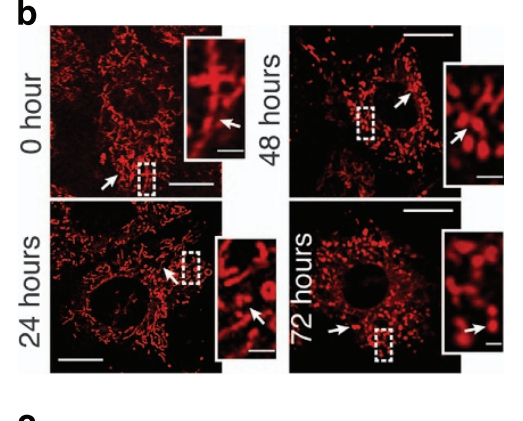

C

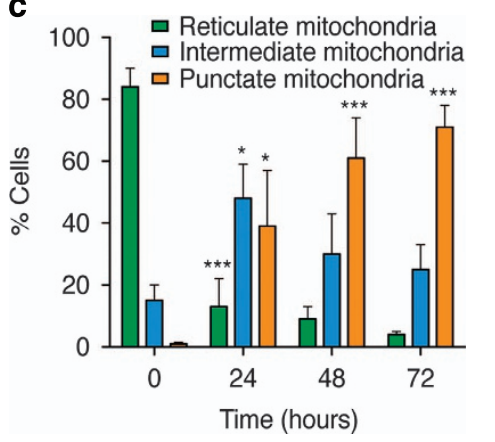

f

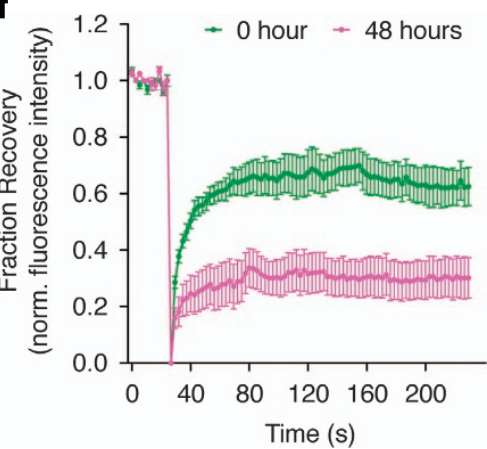

Figure 3 Chronic exposure to hypertrophic agonists compromises $\Delta \Psi \mathrm{m}$. H9c2 cardiomyocytes treated with $100 \mu \mathrm{M} \mathrm{PE}$ or $200 \mathrm{nM}$ Ang II or $10 \mu \mathrm{M}$ Iso for $72 \mathrm{~h}$ were analyzed for $\Delta \Psi \mathrm{m}$ and dynamics in response to hypertrophic stimuli. (a) Confocal micrographs: JC-1 staining. Green channels display mitochondria with compromised $\Delta \Psi \mathrm{m}$ and red channels show mitochondria with positive $\Delta \Psi \mathrm{m}$. Right panels: cytofluorogram. Total magnification $\times 630$. Scale bar, $30 \mu \mathrm{m}$. Inset: Overlay of a single cell displaying mitochondrial heterogeneity of $\Delta \Psi \mathrm{m}$. Scale bar, $5 \mu \mathrm{m}$. (b) Confocal micrographs: mitochondrial morphology. Total magnification $\times 630$. Scale bar, $10 \mu \mathrm{m}$. Inset shows magnified view of the dotted box. Scale bar, $5 \mu \mathrm{m}$. Arrowheads, gradual appearance of fragments from branched networks across time periods. (c-e) Percentage of cells with particular mitochondrial morphology (c); variation in the total number of mitochondria per cell (d); form factor (branching) and aspect ratio (length) (e). (f) FRAP assay showing relative mobility of the mitochondria in cells loaded with MitoTracker red. Plot represents normalized fluorescence intensity profiles before, during and after bleaching (recovery fraction) for $n=16$ cells per group

appearance of punctate structures (Supplementary Figure 1C) after $24 \mathrm{~h}$ of PE treatment. Live cell imaging of the chronically treated cells loaded with MitoTracker red further enabled classification of the morphology into reticulate or branched tubular networks, intermediate fragments and punctate structures with reduced mitochondrial connectivity and motility (Supplementary Figure 1D and Movie 3). Accordingly, we quantified three classes of mitochondrial morphology in chronically hypertrophied H9c2 cardiomyocytes (Figure 3b), which displayed progressive mitochondrial fragmentation with duration of PE treatment (Figures $3 c-e$ and Supplementary Figure 1E). Furthermore, Fluorescence recovery after photobleaching (FRAP) assay on mitochondria-targeted RFP (mito-RFP) revealed delayed and diminished recovery of mitochondrial fluorescence in the agonist-treated cells, which suggested that the altered morphological dynamics of mitochondria severely abrogated its connectivity in the hypertrophied cardiomyocytes (Figure 3f).

Chronic hypertrophic stimulation of cardiomyocytes activates intrinsic cell death signaling. Abrogation of $\Delta \Psi \mathrm{m}$ and morphological dynamics are hallmark features of mitochondrial health and cellular susceptibility to apoptosis. ${ }^{36}$ However, the short-term/24 h treatments with PE, Ang II or Iso did not evoke significant apoptotic phenotypes. Apoptosis gets triggered after $48 \mathrm{~h}$ (Supplementary Figure $1 \mathrm{~F}$ and Figures $4 \mathrm{a}$ and $\mathrm{b}$ ), or follows after a 'chronic' treatment period. Chronic treatments for 48 and $72 \mathrm{~h}$ caused significant elevation of 'initiator' and 'executioner' caspases 9 and 3 , respectively. Active caspase- 9 exhibited a more diffuse 

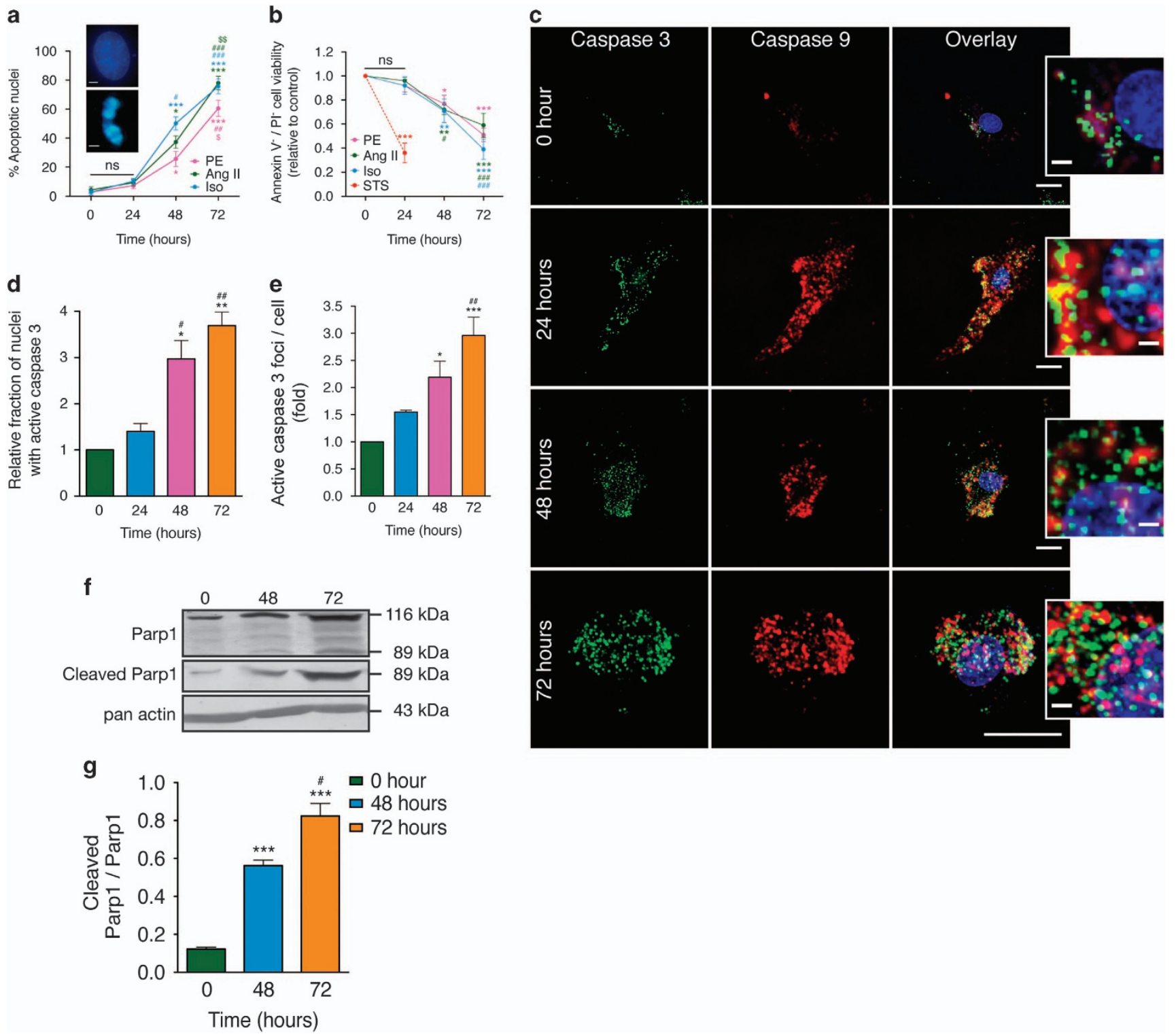

Figure 4 Activation of intrinsic/mitochondrial cell death pathways in response to chronic hypertrophic induction. H9c2 cardiomyocytes treated with hypertrophic agonists were assessed for activation of cell death signaling. (a) Percentage of cells with condensed nuclei; $n=200$ cells per group, ${ }^{* * *, ~}{ }^{\# \# \#} P<0.0001,{ }^{\# \#} P<0.01 ;{ }^{*}, \#, \$ P<0.05$ $\left({ }^{*}, t=0 ;{ }^{*}, t=24 ; \$, t=48, \mathrm{NS}=\right.$ not significant). Inset, normal (top) and apoptotic (bottom) nuclei. (b) H9c2 cell viability in response to chronic hypertrophic treatments.

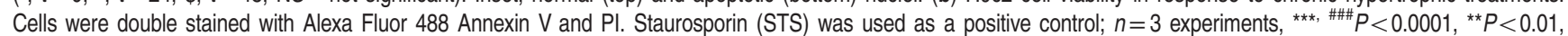
${ }^{*}, \# P<0.05$ ( ${ }^{*}, t=0$; \#, $t=24$; NS = not significant). (c) Confocal micrographs: distributions of active caspases 3 (green) and 9 (red), as revealed by immunostaining with antibodies against cleaved caspases 3 and 9 . Total magnification $\times 600$. Scale bar, $10 \mu \mathrm{m}$. Insets, magnified view of nuclear/perinuclear compartment showing distribution of active caspase signal. Arrowheads, caspase-3 foci. Scale bar, $2 \mu \mathrm{m}$. (d and e) Plots showing the fraction of nuclei with active caspase-3 (d) and the fraction of active caspase-3 foci per nucleus (e) during PE treatment; $n=50,{ }^{++,, * \star *} P<0.0001$; ${ }^{* \star *}{ }^{\# \#} P<0.01 ;{ }^{*} P<0.05\left({ }^{*}, t=0 ;{ }^{\#}, t=24\right)$. (f) Immunoblot showing protein levels of Parp1 and cleaved Parp1 in WCL prepared at the indicated time points after PE treatment. Pan-actin was used as a loading control. (g) Ratiometric quantification of Parp1 activity by densitometry analysis of blots shown in (g); $n=3,{ }^{* *} P<0.0001,{ }^{\#} P<0.05\left({ }^{*}, t=0 ; \#, t=24\right)$

phenotype throughout the cell, whereas caspase-3 was more punctate with distinct foci in the nuclear/perinuclear compartments (Figures $4 \mathrm{c}$ and e). Nuclear accumulation of caspase-3 has been described as an indicator of apoptotic progression, where it is known to cause Parp1 cleavage and subsequent late-stage apoptotic effects. ${ }^{37}$ Immunoblot analysis from cells chronically treated with $\mathrm{PE}$ revealed increased Parp1 activation, denoted by increased $89 \mathrm{kDa}$ cleaved Parp1 fragments (Figures $4 \mathrm{f}$ and $\mathrm{g}$ ).
Anxa6 knockdown abrogates mitochondrial dynamics in chronic cardiomyocyte hypertrophy. Chronic PE treatment of the $\mathrm{H} 9 \mathrm{c} 2$ cardiomyocytes progressively concentrated a sub-population of Anxa6 to mitochondria, as revealed by increased colocalization of Anxa6 (immunolabeling) with mito-RFP (Figures $5 a$ and b). This was further supported by increased protein levels of Anxa6 in the immunoblot analysis of mitochondrial fractions at the indicated time points (Figures $5 \mathrm{c}$ and $\mathrm{d}$ ). To gain mechanistic insight into the 
a
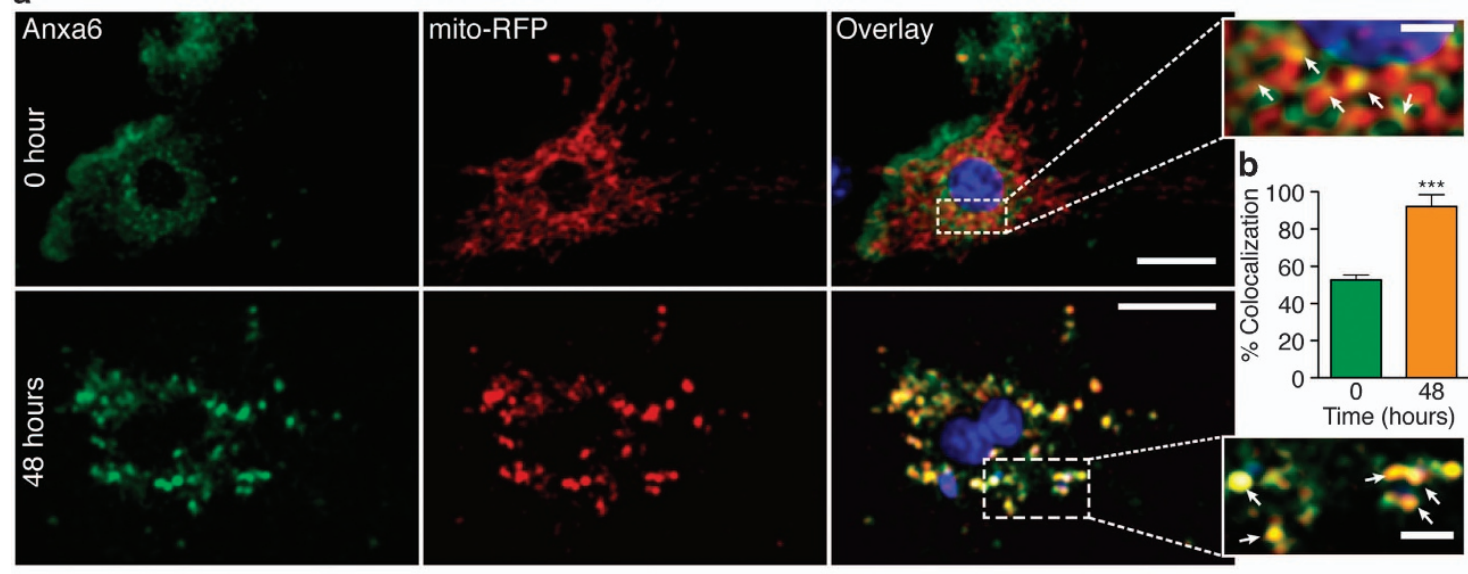

C
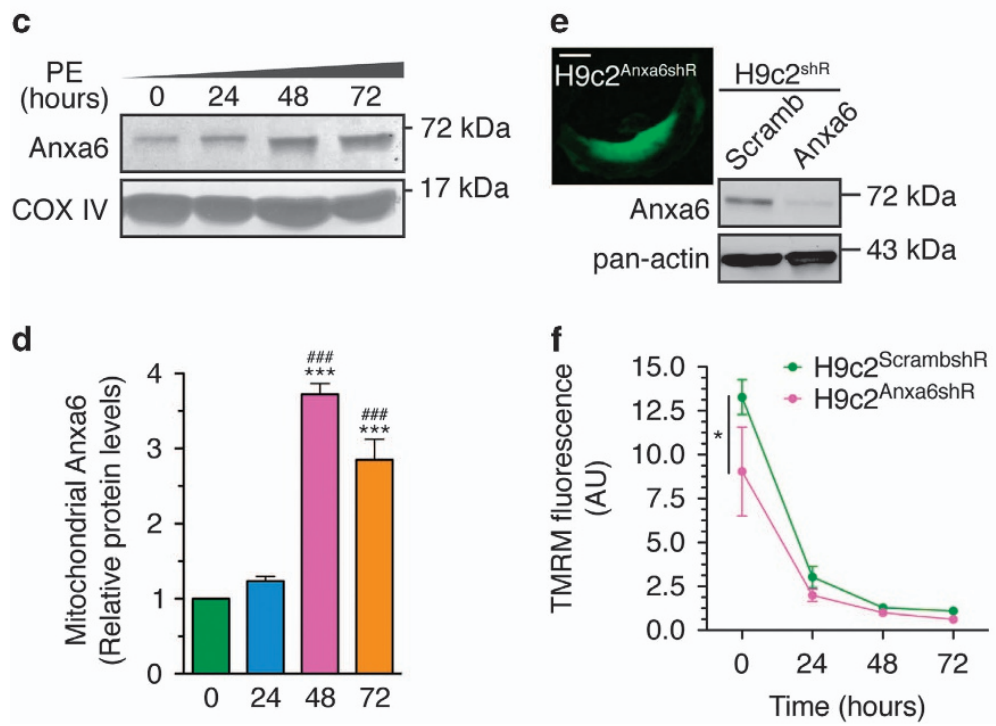

Figure 5 Increased mitochondrial localization of Anxa6 in cardiomyocytes protects against loss of mitochondrial dynamics under chronic hypertrophic induction. H9c2 cardiomyocytes treated with PE were analyzed for Anxa6-mitochondria association and mitochondrial dynamics. (a) Confocal micrographs of cells immunostained for Anxa6 (green) and mito-RFP (red). Nuclei counterstained with DAPI (4',6-diamidino-2-phenylindole; blue). Total magnification $\times 600$. Scale bar, $10 \mu \mathrm{m}$. Insets, colocalized clusters (yellow pixels). Scale bar, $2 \mu \mathrm{m}$. Arrowheads, yellow overlapped pixels. (b) Percentage of colocalization from (a); $n=25$ cells; ${ }^{* * *} P<0.0001$. (c) Immunoblot: protein levels of Anxa6 in mitochondrial fraction from cells treated with PE for indicated time periods. COX IV was used as a loading control for mitochondrial fraction. (d) Quantification of blot in (c), showing Anxa6 protein levels from mitochondrial fractions. Fold changes in PE over $t=0 ; n=3$; ${ }^{* * *}{ }^{\# \# \# ~} P<0.0001$. (e) Immunoblot: Anxa6 knockdown in stable H9c2 cell lines ( $\left.\mathrm{H} 9 \mathrm{c} 2^{\mathrm{shR}}\right)$ for Anxa6 shRNA (H9c2 $\left.{ }^{\text {Anxa6shR }}\right)$, compared with scrambled $\left(\mathrm{H} 9 \mathrm{c} 2^{\text {ScrambshR }}\right)$. Pan-actin was used as a loading control. Inset, a typical H9c2 $2^{\text {Anxa6shR }}$ cell expressing tagGFP as a marker of transfection. Magnification $\times 600$. Scale bar, $10 \mu \mathrm{m}$. (f) TMRM-stained H9c2 ${ }^{\text {ScrambshR }}$ (Scramb) and H9c2 ${ }^{\text {Anxa6shR }}$ (Anxa6) cells. Magnification, $\times 630$. Scale bar, $20 \mu \mathrm{m}$. (g) TMRM fluorescence levels measured from cells shown in (f); $n=50$ cells, ${ }^{\star} P<0.05$

mitochondrial localization of Anxa6 in hypertrophied cardiomyocytes, the status of $\Delta \psi \mathrm{m}$ and dynamics were assessed under Anxa6-knockdown condition, using H9c2 cardiomyocytes stably expressing Anxa6 shRNA (H9c2 ${ }^{\text {Anxa6shR }}$ ) or scrambled control shRNA ( $\mathrm{H} 9 \mathrm{c}^{\text {ScrambshR }}$ ) (Figure $5 \mathrm{e}$ ). Anxa6 knockdown significantly abrogated mitochondrial $\Delta \Psi \mathrm{m}$ over scrambled control, as reveled by the diminished TMRM fluorescence across the period of PE treatment (Figure 5f). Furthermore, such chronic PE treatment severely affected mitochondrial motility (Supplementary Movie 4). Anxa6 knockdown cells have profuse fragmented mitochondria even in the absence of PE treatment and the percentage of fragmented mitochondria gradually increased with the duration of PE treatment (Figures 6a and b). Intriguingly, in spite of the negative effects on mitochondrial dynamics, Anxa6 knockdown seemed to have little effect on the rate of apoptosis in the hypertrophied cardiomyocytes (Figure 6c), suggesting alternative mechanisms in regulating the transition of chronic hypertrophic states towards apoptosis. However, H9c2 ${ }^{\text {AnxabshR }}$ cells exhibited further reduced mitochondrial connectivity after $48 \mathrm{~h}$ of PE treatment compared with the H9c2 ${ }^{\text {ScrambshR }}$ cells, as measured by FRAP assay (Figure 6h).

Ectopic expression of Anxa6 rescues mitochondrial dynamics in hypertrophied cardiomyocytes. To confirm the functions of Anxa6 in the regulation of mitochondrial dynamics and apoptosis in chronically hypertrophied 
a
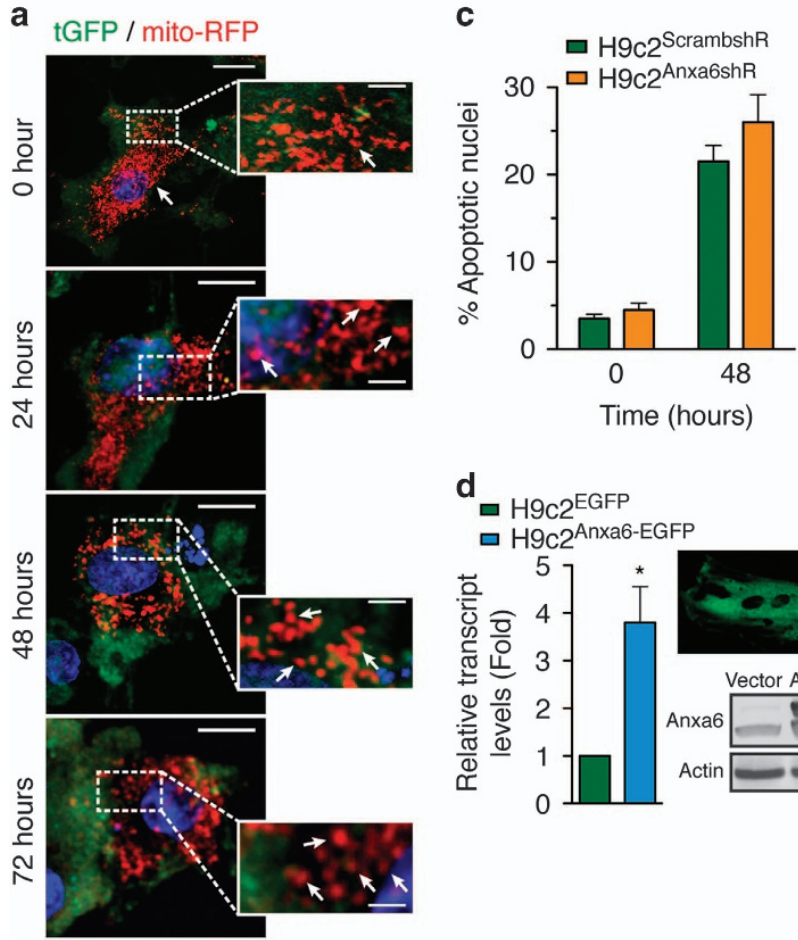

Time (hours)

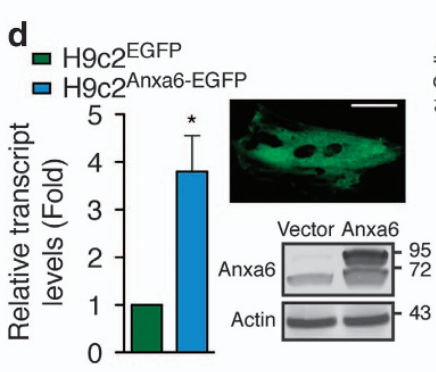

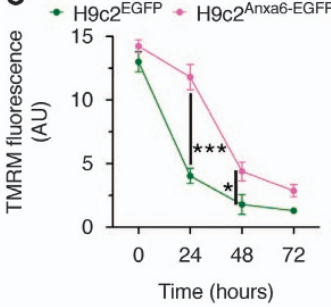

g
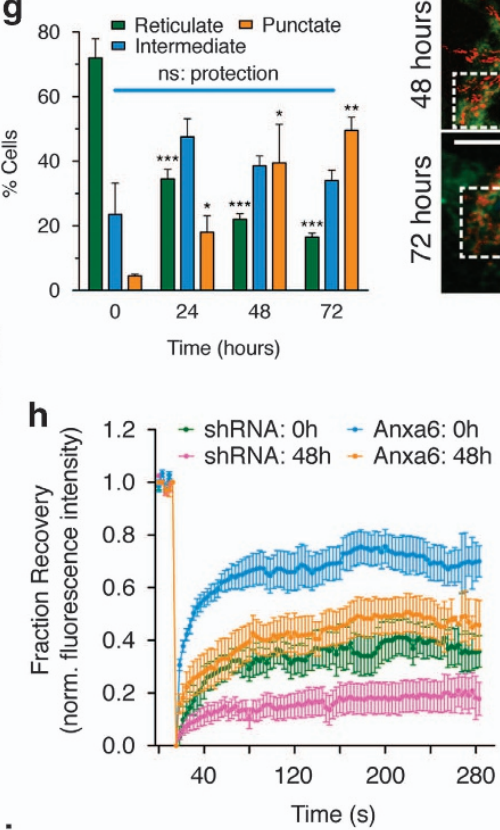
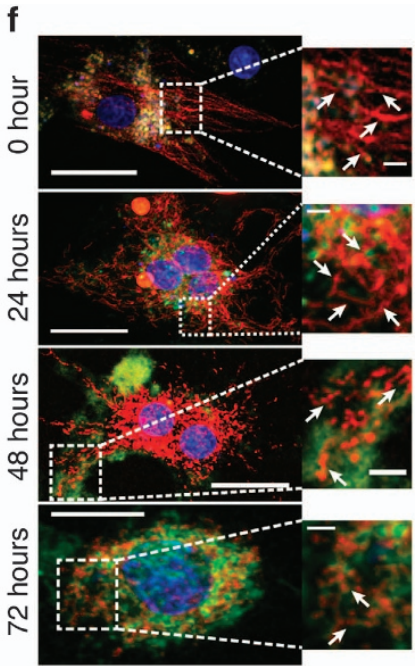

j
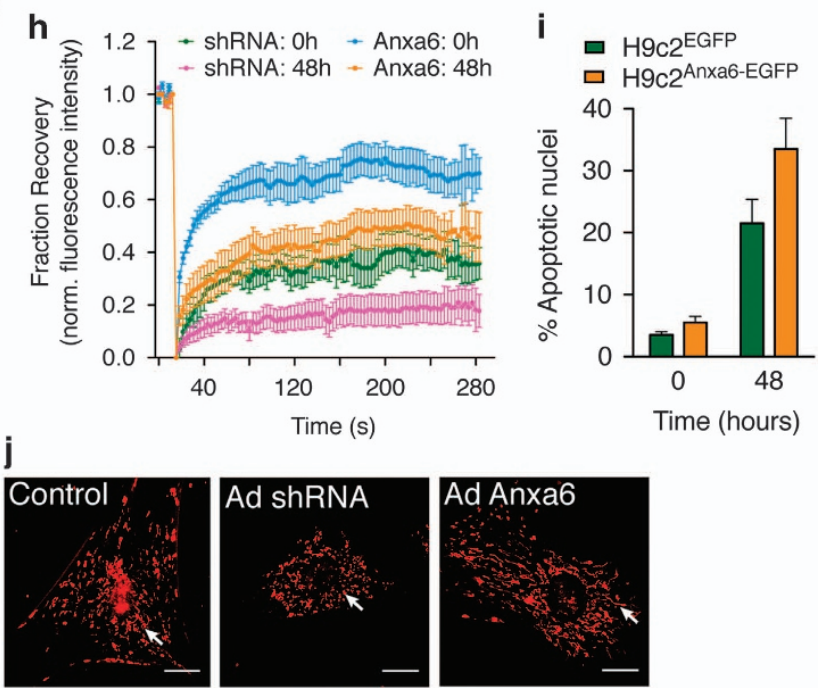

Figure 6 Anxa6 protects mitochondrial dynamics but increases cellular susceptibility to apoptosis. Stable cell lines with downregulated Anxa6 (H9c2 ${ }^{\text {Anxa6shR }) ~ o r ~ e c t o p i c a l l y ~}$ expressing a controlled level of Anxa6 (H9c2 ${ }^{\text {Anxa6-EGFP }}$ ) were treated with PE and analyzed for the effects of Anxa6 on mitochondria and programmed cell death. (a) Confocal micrographs: mitochondrial morphology in H9c2 Anxa6shR cells expressing mito-RFP. Nuclei: Hoechst 33342. Total magnification $\times 600$. Scale bar, $10 \mu \mathrm{m}$. Insets, highlighted boxes. Arrowheads, mitochondrial puncta. Scale bar, $5 \mu \mathrm{m}$. (b) Percentage of cells with reticulate, intermediate or punctate mitochondrial morphology in $\mathrm{H}_{9 \mathrm{c} 2^{\mathrm{Anxa}} \mathrm{ashR}}$ cells; $n=50$, ${ }^{* \star *} P<0.0001$; ${ }^{* \star} P<0.01 ;{ }^{*} P<0.05$ (with respect to $t=0$ ). (c) Percentage of H9c2 ${ }^{\text {Anxa6shR }}$ cells with apoptotic nuclei; $n=200$ cells per group. (d) Fold changes of Anxa6 transcript levels in H9c2 ${ }^{\text {Anxa6-EGFP }}$ cells; $n=3 ;{ }^{*} P<0.05$. Insets, $\mathrm{H} 9 \mathrm{c} 2^{\text {Anxa6-EGFP }}$ cell expressing Anxa6-EGFP. Magnification $\times 630$. Scale bar, $10 \mu \mathrm{m}$ (upper panel). Immunoblot: $\mathrm{H} 9 \mathrm{c} 2^{\text {Anxa6-EGFP }}$ cells with ectopic expression of Anxa6-EGFP fusion. Actin was used as a loading control. (e) $\Delta \Psi \mathrm{m}$-dependent changes in TMRM fluorescence in H9c2 ${ }^{\text {Anxa6-EGFP }}$ cells; $n=3$ experiments; ${ }^{* \star} P<0.0001 ;{ }^{*} P<0.05$. (f) Confocal micrographs: Mitochondrial morphology in H9c2 ${ }^{\text {Anxa6-EGFP }}$ cells. Nuclei: Hoechst 33342 . Total magnification $\times 600$. Scale bar, $10 \mu \mathrm{m}$. Insets, highlighted boxes. Arrowheads, tubular mitochondria, Scale bar, $3 \mu \mathrm{m}$. (g) Percentage of cells with reticulate, intermediate or punctate mitochondrial morphology in H9c2 ${ }^{\text {Anxa6-EGFP }}$ cells; $n=50$; ${ }^{* \star *} P<0.0001 ;{ }^{* \star} P<0.01 ;{ }^{*} P<0.05$ (with respect to $t=0$, NS $=$ not significant); NS, intermediate morphology representing protection. (h) FRAP assay for quantification of relative mobility of mitochondria in H9c2 ${ }^{\text {Anxa6-EGFP }}$ cells infected with mito-RFP baculovirus. Plot showing normalized fluorescence intensity profiles before, during and after photobleaching (recovery fraction) for $n=15$ cells per group. (i) Percentage of H9c2 ${ }^{\text {Anxa6-EGFP }}$ cells with apoptotic nuclei ( $n=3$ experiments with 200 cells per group). (j) Fluorescence micrographs: mitochondrial morphology in NRVMs infected with control, Anxa6 shRNA or FL Anxa6 protein encoding adenovirus and cultured with PE for $48 \mathrm{~h}$

cardiomyocytes, stable $\mathrm{H} 9 \mathrm{c} 2$ cell lines ectopically expressing controlled levels of Anxa6-EGFP (H9c2 ${ }^{\text {Anxa6-EGFP) }}$ or the empty vector ( $\mathrm{H} 9 \mathrm{c} 2^{\mathrm{EGFP}}$ ) were generated (Figure $6 \mathrm{~d}$ ). A controlled expression level ( $\sim$ - to 4.5-fold increase over control) was essential as uncontrolled overexpression of Anxa6 resulted in cell death in our pilot experiments (data not shown) and, as reported by others, leads to the development of cardiomyopathies in animal models. ${ }^{19}$ Analysis of TMRM fluorescence levels indicated that mitochondrial $\Delta \Psi_{\mathrm{m}}$ was significantly more conserved during $P E$ treatment in the H9c2 ${ }^{\text {Anxa6-EGFP }}$ cells compared with the control (H9c2 ${ }^{\text {EGFP }}$ ) cells, especially during the first $24 \mathrm{~h}$ of treatment (Figure $6 \mathrm{e}$ ). Live cell imaging of mitochondria showed H9c2 Anxa6-EGFP cells have extensive tubulature/reticulate mitochondrial 
A

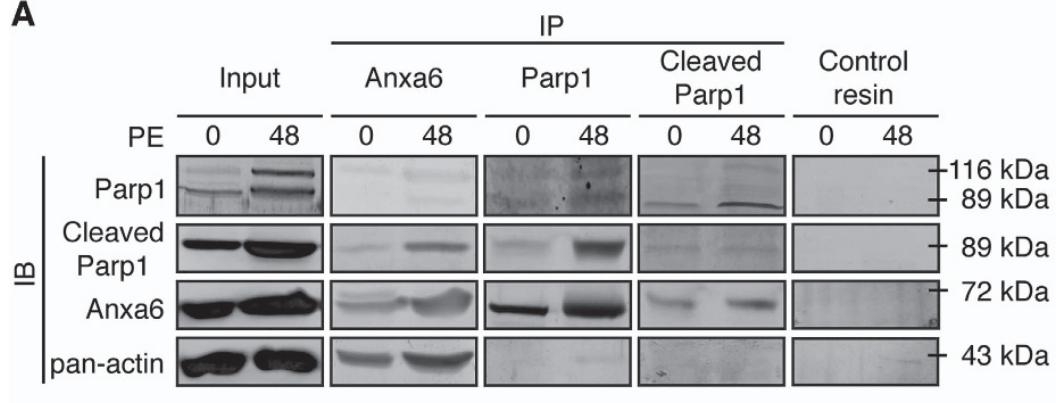

B

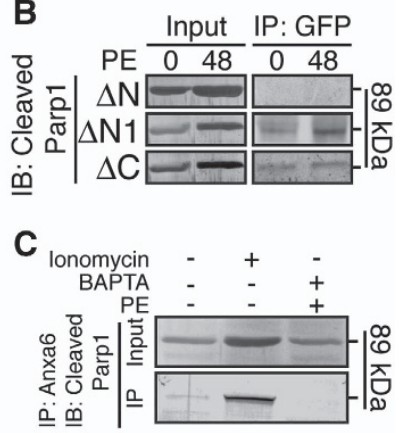

D
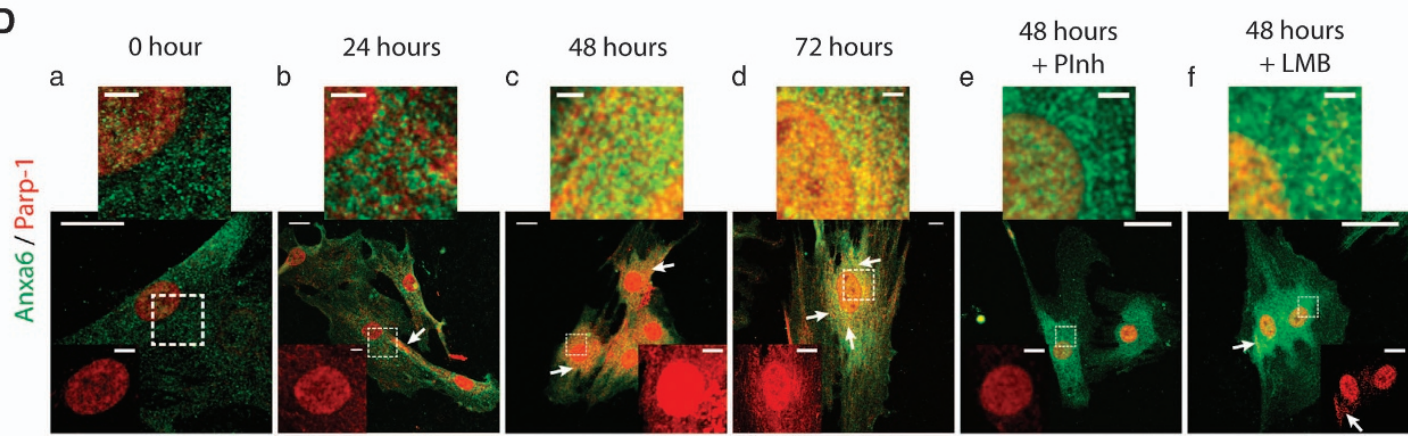

Figure 7 Interactions of Anxa6 with Parp1 in cardiomyocytes. H9c2 cardiomyocytes treated with PE were examined for association between Anxa6 and Parp1. (A) WCL from wild-type H9c2 (input), without, or treated with PE were subjected to IP, followed by immunoblot (IB) analysis of the immunoprecipitate. Antibodies for IP are indicated at the top, and for IB are indicated on the left. Pan-actin was used as an antibody control for IP and a loading control for input IB, respectively. (B) WCL (input) from H9c2 cells expressing Anxa6 deletion mutants indicated on left, after PE treatment, were subjected to IP, followed by IB. Living colors (JL-8) antibody was used to pull-down EGFP fusion (IP) and was probed for IB using antibody for cleaved Parp1. (C) WCL (input) from wild-type H9c2 (input), pretreated with ionomycin or BAPTA and without, or treated with PE for $48 \mathrm{~h}$, were subjected to IP, followed by IB analysis of the Anxa6 immunoprecipitate for cleaved Parp1. (D) Confocal micrographs: cells double immunostained for Anxa6 (green) and Parp1 (red). Cells were treated without (a) or with PE for 24-72 $\mathrm{h}(\mathrm{b}-\mathrm{d}$ ) or in combination with either PARP inhibitor (e) or Leptomycin B (f). Scale bar, $10 \mu \mathrm{m}$. Arrowheads, cytosolic Parp1 signal and colocalized pixels. The magnified image of the white box shows perinuclear compartment with Anxa6-Parp1 distribution. Scale bar, $3 \mu \mathrm{m}$. Inset, nuclear/ perinuclear compartment showing leakage of Parp1 to cytosol. Scale bar, $2 \mu \mathrm{m}$

network, after $24 \mathrm{~h}$ and significantly higher proportion of intermediate fragments after $48 \mathrm{~h}$ of PE treatment (Figures $6 f$ and g). The importance of Anxa6 as an enhancer of mitochondrial dynamics was further evident in mitochondrial connectivity analysis by FRAP assay, which showed significantly higher recovery rates of mitochondrial fluorescence intensity in $\mathrm{H}_{\mathrm{c}} 2^{\mathrm{AnxaG}-\mathrm{EGFP}}$ cells over the control cells (Figure 6h). However, the H9c2 ${ }^{\text {Anxa6-EGFP }}$ cells displayed higher levels of apoptotic nuclei (Figure 6i), which may be indicative of Anxa6 participating in alternate mechanisms.

To further confirm the involvement of Anxa6 in mitochondrial dynamics, hypertrophy was induced in neonatal rat ventricular cardiomyocytes (NRVMs) by PE. Hypertrophy in NRVMs was validated by immunostaining with ANP antibody showing enhanced perinuclear localization of ANP as a marker of hypertrophy after $24 \mathrm{~h}$ of stimulation with PE (Supplementary Figure S1G). Fragmentation of mitochondria was observed with the duration of PE treatment in NRVMs (Supplementary Figure S1H). Mitochondrial morphology was also examined in NRVMs ectopically expressing Anxa6 shRNA or full-length (FL) Anxa6. Consistent with H9c2 cardiomyocytes, mitochondrial fragmentation was also increased in hypertrophied NRVMs. Knockdown of Anxa6 further deteriorated mitochondrial morphology, which was restored almost completely when Anxa6 was overexpressed (Figure 6j).
Anxa6 interacts with Parp1 in hypertrophied cardiomyocytes. Parp1 is known to have vital roles in cardiac remodeling ${ }^{38}$ and is activated (via cleavage) parallel to ANP secretion in response to hypertrophic stimulus. ${ }^{39}$ It is also instrumental in late phases of apoptosis, with Parp1 cleavage by caspases being a hallmark feature. ${ }^{40}$ Although Parp 1 is a nuclear enzyme, the $89 \mathrm{kDa}$ cleaved fragment moves to the cytosol via nucleocytoplasmic transport. ${ }^{41}$ Thus, we questioned whether Anxa6 interacts with Parp1 in H9c2 cardiomyocytes in course of chronic hypertrophy to bring about the differential apoptotic responses observed under ectopic Anxa6 expression. Co-immunoprecipitation (co-IP) experiments showed an increased physical association of Anxa6 with the $F L$ as well as to a greater extent with the cleaved Parp1 under PE treatment (Figure 7A). Furthermore, $\mathrm{N}$-terminal deletion mutants of Anxa6, $\Delta \mathrm{N}(1-89)$ and $\Delta \mathrm{N} 1$ (29-89) exhibited a differential interaction with cleaved Parp1 (Figure $7 \mathrm{~B}$ ), with $\Delta \mathrm{N}$ showing total loss of physical interaction, whereas $\Delta \mathrm{N} 1$ showing interaction similar to the FL. This signifies that the N-terminal residues (1-29) of Anxa6 have a crucial role in regulating its interaction with Parp1. Moreover, C-terminal mutant $\Delta C$ (600-673) showed partial binding with cleaved Parp1, presumably because of abrogated $\mathrm{Ca}^{2+}$ binding. ${ }^{42}$ Treatment of the cells with ionomycin resulted in enhanced deposition of cleaved Parp1 in Anxa6 immunoprecipitate, whereas cells pretreated with the $\mathrm{Ca}^{2+}$-chelator 

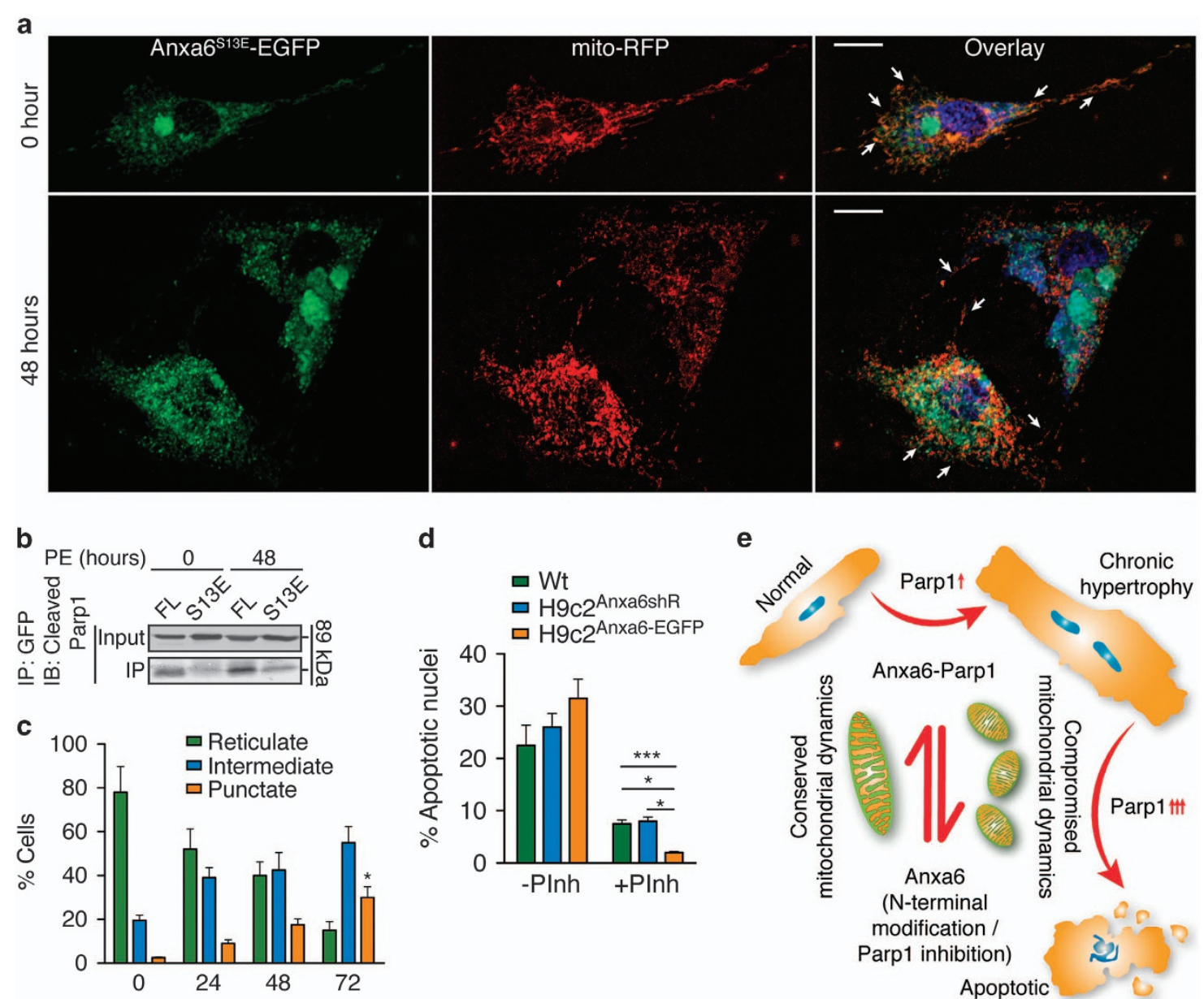

d

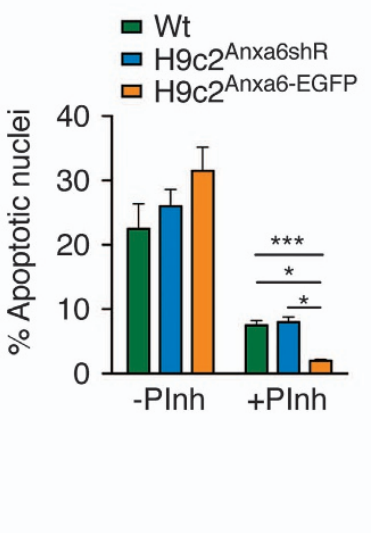

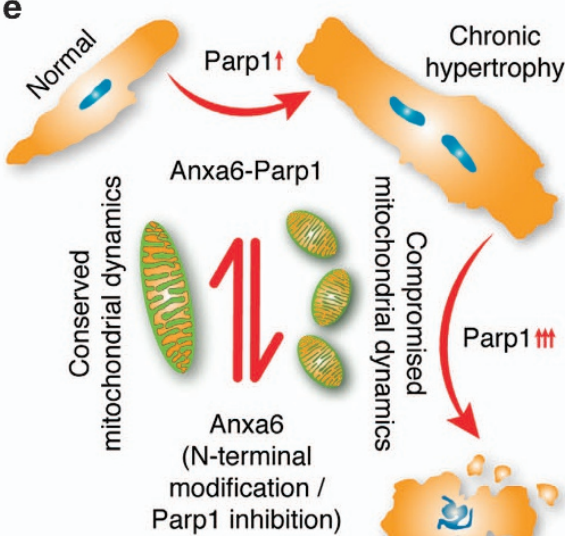

Apoptotic

Figure 8 Anxa6 is essential for conservation of mitochondrial dynamics in hypertrophied cardiomyocytes. H9c2 cardiomyocytes treated with PE were analyzed for the functional involvement of Anxa6 in mitochondrial dynamics and cell death signaling. (a) Confocal micrographs: H9c2 cells transiently transfected with S13E mutant and infected with mito-RFP were treated with PE and analyzed for distribution of S13E with respect to mitochondria. Orange pixels in overlay channel indicate significant colocalization of Anxa6 with S13E. Arrowheads, tubular mitochondria, Scale bar, $10 \mu \mathrm{m}$ (upper panel) and $15 \mu \mathrm{m}$ (lower panel). (b) WCL (input) from H9c2 cells expressing Anxa6FL or Anxa6S13E, after indicated periods of PE treatment, were subjected to IP, followed by immunoblot (IB) analysis of the immunoprecipitate. (c) Percentage of cells with reticulate, intermediate or punctate mitochondrial morphology in $\mathrm{H} 9 \mathrm{c} 2^{\mathrm{Anxa6S13E}}$ cells treated with $\mathrm{PE}$ for indicated periods; $n=50$; ${ }^{*} P<0.05$. (d) Percentage of cells with apoptotic nuclei after $48 \mathrm{~h}$ of PE treatment, without, or pretreated with PInh; $n=3$ experiments with 200 cells per group; ${ }^{* \star \star} P<0.0001 ;{ }^{*} P<0.05$. (e) Schematic depiction of the dual regulatory functions of Anxa6 that mediates transition of hypertrophy to apoptosis and conserves mitochondrial dynamics in hypertrophied cardiomyocytes. Anxa6-Parp1 interaction shifts the equilibrium through chronic hypertrophy and apoptotic states and compromises mitochondrial dynamics, whereas negative regulation of Parp1 activation or modifications in Anxa6 that depletes the interaction, shifts the equilibrium away from cell death and enhances mitochondrial dynamics in hypertrophied cardiomyocytes

BAPTA (1, 2-Bis (2-aminophenoxy) ethane- $N, N, N^{\prime}$, $N^{\prime}$-tetraacetic acid tetrakis (acetoxymethyl ester)) showed complete absence of cleaved Parp1 in Anxa6 co-IP (Figure 7C). Thus, it is evident that chronic hypertrophy of $\mathrm{H} 9 \mathrm{c} 2$ cardiomyocytes is also coupled with a $\mathrm{Ca}^{2+}$-dependent interaction of Anxa6 with Parp1 via its $\mathrm{N}$ terminus.

\section{Anxa6-mediated protection of mitochondrial dynamics} counterbalances its interaction with Parp1. To analyze the functional significance of Anxa6-Parp1 interaction in hypertrophied cardiomyocytes, Parp1 localization was examined in course of PE treatment. Immunofluorescence analysis showed increased cytosolic Parp1 localization through 48-72h. Such cytosolic localization was not observed when cells were pretreated with Parp1 inhibitor (PInh) or nuclear export inhibitor leptomycin B (LMB)
(Figure 7D). It demonstrated that Parp1 activation is required for the nucleocytoplasmic transport. The co-IP results from the mutants suggested that the first 30 residues of Anxa6 seemed to be vital for the physical association with Parp1, which includes a putative phosphorylation site (S13). We found that the mutant Anxa6 ${ }^{\mathrm{S} 13 \mathrm{E}}$ exhibited enhanced mitochondrial localization (Figure 8a) and reduced interaction with cleaved Parp1 (Figure 8b). The H9c2 ${ }^{\text {Anxa6-S13E }}$ cells displayed enhanced mitochondrial tubulature even after $48 \mathrm{~h}$ of PE treatment (Figure $8 \mathrm{c}$ ). It appears that the association of Anxa6 with Parp1 mediates activation of the later; an event that may contribute to elevated apoptotic counts in Anxa6-expressing hypertrophied cells. Treatment of the wild-type, $\mathrm{H} 9 \mathrm{c} 2^{\text {Anxa6-shR }}$ or $\mathrm{H} 9 \mathrm{c} 2^{\text {Anxa6-EGFP }}$ cells with the PInh significantly reduced the number of apoptotic nuclei (Figures 8d and e). Moreover, treatment with the inhibitor 
restored mitochondrial tubulature and motility in Anxa6knockdown cells (Supplementary Movie 6), indicating a direct role of Anxa6/Parp1 in the death pathway.

\section{Discussion}

Signaling cross-talks drive several pathophysiological conditions in mammalian cells. The spatiotemporal dynamics of signaling molecules with the subcellular compartments are crucial for efficient signal dispersal. ${ }^{43-45}$ The present work describes such a regulatory circuit in the chronic hypertrophy of cardiomyocytes, in which Anxa6 helps in the conservation of equilibrium between hypertrophy and apoptosis. These conclusions are based on gain- and loss-of functions approaches using $\mathrm{H} 9 \mathrm{c} 2$ cardiomyocytes, where we characterize the phenotypes associated with hypertrophied signaling and incorporate the functions of Anxa6 in the hypertrophic process.

Here, the induction of hypertrophy by PE, Ang II or Iso for $48-72 \mathrm{~h}$ is described as 'chronic' as the phenotypes at the cellular level are robust and exhibit signs of maladaptive stress response and deteriorating cell health, in comparison with treatments for $24 \mathrm{~h}$. Coupled with enlarged cell size and expression of hypertrophy markers, upregulated expression of Anxa6 is observed along with its cellular relocalization (Figures 1 and 2). As described earlier, ${ }^{24}$ Anxa6 forms punctate aggregates in hypertrophied cardiomyocytes. Interestingly, the punctate structures are distributed in the perinuclear compartment in the later phases of stimulation (Figure 1; 48-72 h).

Anxa6 has been implicated in the regulation of mitochondrial morphology in fibroblasts. ${ }^{17}$ Prolonged stimulation of cardiomyocytes with the hypertrophic agonists resulted in a drop of mitochondrial $\Delta \psi \mathrm{m}$ (Figure $3 \mathrm{a}$ and Supplementary Figure 1). Loss or collapse of the mitochondrial $\Delta \Psi \mathrm{m}$ is known to affect negatively the fused reticulate network of mitochondria, increasing the fission cycle. A dynamic balance between mitochondrial fusion and fission is crucial for the maintenance of homeostasis and a major determinant in driving the cell state towards apoptosis. ${ }^{46-48}$ Further examinations on whether such loss of $\Delta \psi_{\mathrm{m}}$ causes mitochondrial punctation or loss of dynamics reveal the presence of fragmented mitochondria in PE-treated cells. Such fragmentation patterns, as also described by others, ${ }^{49}$ are clearly evident after PE treatment. Loss of mobility and connectivity of the organelle are evident in the live cell imaging analysis and was further quantified by FRAP assay (Figure 3 and Supplementary Figure 1 and Supplementary Movie 3). Such analysis clearly shows an increased recovery period and decreased mobile fraction in the hypertrophied cells. Thus, it is evident that chronic hypertrophy of cardiomyocytes accelerates the mitochondrial fission cycle.

The mitochondrial pathway of cell death is activated in response to the loss of mitochondrial dynamics in cardiomyocytes, ${ }^{50}$ and recently, the intrinsic caspase activation cascade is reported to be instrumental for the development of cardiac hypertrophy. ${ }^{34}$ However, whether such developments culminate to apoptotic cell death remains controversial. Furthermore, apoptotic phenotypes associated with transition of hypertrophy to heart failure ${ }^{7}$ does not faithfully reproduce in agonist-induced hypertrophy of isolated cardiomyocytes in culture and the available reports are contradictory. $^{3,51-55}$ This severely limits our understanding of the intricate regulatory mechanisms governing each phenomenon. Here, we characterize the programmed cell death signaling activation in course of chronic hypertrophy as a 'mild' phenotype that elevates with longer periods of treatment. The different agonists exhibit differential behavior in their potency to induce apoptosis in our studies (Figures $4 \mathrm{a}$ and $\mathrm{b}$ ), thus revealing pathway preferences in progression of hypertrophy towards apoptosis. Specifically, PE is found to be more protective in comparison with Iso or Ang II. However, activations of both 'initiator' and 'executioner' caspases 3 and 9 , respectively, are observed in $\mathrm{H} 9 \mathrm{c} 2$ cardiomyocytes chronically treated with PE (Figures $4 \mathrm{c}-\mathrm{e}$ ), which progress towards Parp1 cleavage, a hallmark of the late-phase apoptosis (Figures $4 \mathrm{f}$ and $\mathrm{g}$ ). The discrepancy in activation of apoptotic machinery at the molecular levels and their execution at the cellular level could be explained by the presence of a dynamic equilibrium consisting of the counterbalancing feedback circuitry that shifts the equilibrium to a direction favorable for the maintenance of cellular homeostasis. ${ }^{3}$ As Anxa6 has multiple scaffolding functions, it appears to be a promising candidate for synchronizing such machineries.

Chronic PE treatment induces progressive association of Anxa6 with mitochondria (Figure 5), which is essential for preventing loss of mitochondrial dynamics in hypertrophy, as Anxa6 knockdown cells display severely abrogated mitochondrial $\Delta \Psi \mathrm{m}$ and dynamics (Figures $5 \mathrm{f}$ and $6 \mathrm{a}, \mathrm{b}$; Supplementary Movie 4). However, ectopically expressed Anxa6 at controlled levels protects the cardiomyocytes against such loss of mitochondrial dynamics (Figures $6 \mathrm{e}$ and $\mathrm{g}$ and Supplementary Movie 5). This pattern is more evident in the FRAP analysis of mitochondrial connectivity, which displays increased mobile fraction and decreased recovery time in Anxa6-expressing cells (Figure 6h). It demonstrates that the presence of Anxa6 provides a significant level of protection against loss of mitochondrial dynamics in hypertrophied cardiomyocytes. However, subtle differences are observed in apoptotic nuclear morphology in $\mathrm{H} 9 \mathrm{c} 2^{\text {Anxa6-EGFP }}$ and $\mathrm{H} 9 \mathrm{c} 2^{\text {Anxa6shR }}$ cells (Figures $6 \mathrm{c}$ and i), compared with controls, with Anxa6-expressing cells having relatively higher levels of apoptotic cells at $48 \mathrm{~h}$. Thus, we hypothesize that Anxa6 might be interfering with components of the dynamic equilibrium between hypertrophy and apoptosis in cardiomyocytes.

We have shown earlier that Parp1 interacts with Anxa6 in the myocardium. ${ }^{23}$ Here, we found that both Parp1 and its $89 \mathrm{kDa}$ cleaved fragment physically bind with Anxa6 in PE-treated cardiomyocytes (Figure 7). Consistent with the earlier report, Parp1 activation in cardiomyocytes is found to be a $\mathrm{Ca}^{2+}$-dependent process. ${ }^{39}$ A progressive increase in the cytosolic signal of Parp1 was observed with increasing treatment periods, which is totally abolished by inhibition of Parp1 or nuclear export (Figure 8d), suggesting that the activation of Parp1 is instrumental for the process.

The N-terminal residues (1-29) of Anxa6 that seems crucial for interacting with Parp1 also contains a putative phosphorylation site at $\mathrm{S} 13 .{ }^{12}$ To examine the possible roles of the posttranslational modification of Anxa6 in regulating its 
dynamics, the phosphomimic S13E mutant is used, which displays enhanced mitochondrial localization (Figure 8a). Binding of Parp1 with Anxa6 is abrogated in cells expressing the S13E mutant (Figure 8b). Surprisingly, the mitochondrial localization of Anxa6 is enhanced in these cells, which may be responsible for improved mitochondrial dynamics in Anxa6 ${ }^{\mathrm{S} 13 \mathrm{E}}$ cardiomyocytes (Figure $8 \mathrm{c}$ ). In other words, Anxa6 ${ }^{\mathrm{S} 13 \mathrm{E}}$ technically improves the functionality of Anxa6 to act as an enhancer of mitochondrial dynamics, bypassing the Parp1-mediated shift of the equilibrium towards apoptotic state. Such counterbalancing mechanism of Anxa6 function was clearly visualized in $\mathrm{H} 9 \mathrm{c} 2^{\text {Anxa6shR }}$ cells after PE treatment and pretreatment with PInh. These cells displayed abundant mitochondrial motility and enriched intermediate fragments (Supplementary Movie 6).

To our knowledge, the present study is the first evidence where spatiotemporal dynamics of Anxa6 is shown to be critical for maintaining equilibrium between chronic hypertrophy and cell death to avoid stressful maladaptations of cardiomyocytes. However, the in vitro analysis of mitochondrial dynamics and cell death using experimental model of H9C2 cardiomyocytes remain a limitation of this study and whether such mechanisms operate in vivo warrants further investigation. In summary, we have uncovered a dual regulatory role of Anxa6, one that regulates Parp1 activation and subsequent cell death machineries and the other as an enhancer of tubular mitochondrial morphology in hypertrophied cardiomyocytes, thereby acting as a molecular switch that modulates the transition of hypertrophic phase to apoptosis. However, the former role, as described above, depends on a multitude of signaling mediators and demands further characterization. As mitochondrial dynamics is emerging as a potential new therapeutic target for heart failure, ${ }^{56}$ the scaffolding activity offered by Anxa6 holds much promise as a positive regulator of mitochondrial dynamics in hypertrophied cardiomyocytes.

\section{Materials and Methods}

Reagents. Common laboratory reagents were purchased from Life Technologies (Grand Island, NY, USA), Sigma (St. Louis, MO, USA) and Thermo Scientific (Waltham, MA, USA), unless otherwise mentioned. PE, Ang II, Iso and LMB were from Sigma. Ionomycin, BAPTA-AM, fluorescent conjugates and other microscopy consumables were from Life Technologies. Mitochondria Isolation Kit and Co-IP Kits were from Pierce Biotechnology (Rockford, IL, USA). PInh was from Calbiochem (La Jolla, CA, USA). JC-1 Staining Kit was from Cayman Chemicals (Ann Arbor, MI, USA). DAPI, Hoechst 33342, propidium podide (PI), Annexin V-Alexa Fluor 488, TMRM, MitoTracker red FM, CellLight Mitochondria-RFP and BacMam 2.0 system were from Life Technologies. Primary antibodies were procured from the following sources: Anxa6 (monoclonal antibody) from BD (Lexington, KY, USA); Anxa6 (polyclonal antibody), COX IV (cytochrome $c$ oxidase subunit IV), Akt, p-AktS473 and $\alpha$-SkA from Santa Cruz Biotechnology (Santa Cruz, CA, USA); $\alpha$-tubulin, cleaved caspase-3, cleaved caspase-9, Parp1 and cleaved Parp1 from Cell Signaling Technology (Beverly, MA, USA); pro-ANP from Abcam (Cambridge, MA, USA); anti-COX I monoclonal antibody from Invitrogen (Carlsbad, CA, USA); Living Colors anti-fluorescent protein antibody (JL-8) from Clontech (Mountain View, CA, USA) and pan-actin from Chemicon (Temecula, CA, USA)

Constructs. Plasmid vectors and Anxa6 shRNA constructs were acquired from Clontech and OriGene Technologies (Rockville, MD, USA), respectively. Generation of the EGFP-tagged Anxa6 constructs has been described earlier. ${ }^{24}$ Site-directed mutants of Anxa6 were generated using the Quik Change Lightning Kit (Stratagene, La Jolla, CA, USA) following the manufacturer's instructions. Primers used for construct generation and site-directed mutagenesis were described earlier. ${ }^{24}$
For generation of the mutant Anxa6S13E, the following pairs of mutagenic primers were used: (forward) 5'- GTGCCATGTACCGAGGCGAGGTCCACGACTTCGCAGA-3' and (reverse) 5'-TCTGCGAAGTCGTGGACCTCGCCTCGGTACATGGCAC-3'. To knockdown Anxa6 in NRVMs, Anxa6 shRNA was prepared and the adenoviral vectors (kindly provided by B Vogelstein, The Johns Hopkins Medical Institutions, Baltimore, MD, USA) expressing shRNAs were generated following the guidelines for efficient siRNA designing protocol. ${ }^{57}$ For ectopic expression of FL Anxa6, adenoviral construct was prepared using the following primers: (forward) 5'-ACTTAAGCTTCGAGCTGCGTCTATCTGTCTGC-3' and (reverse) 5'-CGAG TCTAGAGCACCCCAGCCGGATCACAGT-3',

Cell culture. H9c2 cardiomyocytes acquired from the National Centre for Cell Science (Ganeshkhind, Pune, India) were cultured in DMEM with high glucose $(4.5 \mathrm{~g} / \mathrm{l})$, sodium bicarbonate $(1.5 \mathrm{~g} / \mathrm{l})$ and FBS $(10 \%)$ in a incubator at $37^{\circ} \mathrm{C}, 5 \%$ $\mathrm{CO}_{2}$ and $80 \% \mathrm{RH}$. Cells were serum starved for $18-24 \mathrm{~h}$ before experimentation. Transfection, time frames for analysis of gene expression and generation of stable cell line have been described before. ${ }^{24}$ The Anxa6S13E mutant took more time in maturation and expression posttransfection. Consequently, experimentation involving Anxa6S13E was carried out $60-72 \mathrm{~h}$ posttransfection. Baculoviral mito-RFP transduction was carried out as per the manufacturer's protocol. Briefly, imaging assays were setup 24-28 $\mathrm{h}$ after the addition of virus. Hypertrophy was induced by treating serum-starved cells with $100 \mu \mathrm{M}$ PE or $300 \mathrm{~nm}$ Ang II or $10 \mu \mathrm{M}$ Iso for indicated time periods. For ionomycin and BAPTA treatments, cells were preincubated with either $4 \mu \mathrm{M}$ of ionomycin for a period of 10 min or with $10 \mu \mathrm{M}$ of BAPTA-AM for a period of 30 min. Pretreatment with PInh XIV was carried out with $10 \mu \mathrm{M}$ for $30 \mathrm{~min}^{58} \mathrm{NRVMs}$ from 2- to 3-day-old Sprague-Dawley rat pups were isolated as described earlier. ${ }^{59,60}$

Real-time quantitative RT-PCR. The following primers were used for quantitative real-time RT-PCR: Anxa6 (forward) 5'-GCCGCTTGCCTATTGTGAC-3', Anxa6 (reverse) 5'-GCTGGTGTATCTGCTCATTGG-3'; $18 \mathrm{~S}$ rRNA (forward) 5'-CGGCTACCACATCCAAGGAA-3', $18 \mathrm{~S}$ rRNA (reverse) 5'-AGCTGGAATTACC GCGGC-3'. Relative quantification of gene expression was performed as described earlier. ${ }^{28,61}$ Analysis of the fold changes in transcript profiles by $2^{-\Delta \Delta C T}$ method has been described elsewhere. ${ }^{62}$ Fold changes in the mRNA levels were normalized to $18 \mathrm{~S}$ rRNA levels.

Fractionation, co-IP and western blotting. Whole-cell lysates (WCL) were made in Celllytic MT buffer (Sigma) and were supplemented with Halt proteasephosphatase inhibitor cocktail (Thermo Scientific Pierce, Waltham, MA, USA). Mitochondrial fractionation and co-IP experiments were carried out using commercially available kits (Pierce) as per the manufacturer's instructions. Briefly, $5 \times 10^{6}$ cells were used for mitochondrial isolation and pelleted mitochondria were boiled in lysis buffer for $3 \mathrm{~min}$ to yield mitochondrial lysate. For co-IP experiments, $1 \mathrm{mg}$ of the lysate (made with the supplied IP lysis buffer with added Halt protease and phosphatase inhibitor mixture) was subjected to IP, with $10 \%$ of the starting material $(\sim 100 \mu \mathrm{g})$ used as an input. Antibodies were coupled onto amine-reactive resins and the lysates were incubated with these antibody-coupled resins or control resins provided with the kit overnight at $4{ }^{\circ} \mathrm{C}$ with end-to-end rotation to ensure homogeneous mixing. About $25-$ $100 \mu \mathrm{g}$ of the elution fraction was subjected to the subsequent western blot analysis. SDS-PAGE, blotting and the detection methods with subsequent densitometric quantification were performed as described earlier. ${ }^{28,61}$

Microplate assays. All microplate assays were carried out aseptically in Special Optics black-walled 96-well plates (Corning Coster, Tewksbury, MA, USA) to reduce well-to-well cross-talks and improve the signal-to-noise ratio of data acquisition. Signal detection was carried out in a Spectramax Paradigm multimode detection platform (Molecular Devices, Sunnyvale, CA, USA) with dynamic z-stepper, operating in bottom-read mode.

Microscopy. Confocal microscopy and live cell imaging setups and the procedure for immunocytochemistry (ICC-IF) have been described earlier. ${ }^{24}$ High-resolution image acquisition was performed by slow unidirectional sequential scanning (200-400 Hz; beam expander: 6) and smaller frame sizes were used for higher magnification. For time-lapse imaging, faster scanning speeds $(800-1000 \mathrm{~Hz})$ coupled with low line averaging $(0-2)$ were used to preserve temporal resolution. Pixel dimensions and Z-steps were estimated to satisfy Nyquist sampling criteria. Disc spinning speed of the CSU was set to 1800 r.p.m. All comparable sets of images were acquired under identical stack parameters, laser 
power, detector gain, offset and pinhole aperture windows. Staining of cells with phalloidin, TMRM, JC-1, Hoechst and MitoTracker red were performed as per the manufacturer's instructions. Live cell imaging of mitochondria was performed as described elsewhere. ${ }^{63}$ FRAP assay was carried out using an S APO $63.0 \times 1.42 \mathrm{NA}$ oil objective at room temperature $\left(22^{\circ} \mathrm{C}\right)$. One hundred percent of a $561 \mathrm{~nm}$ laser line was used for bleaching a small rectangular region of interest in a single scan. Recovery of fluorescence was then monitored at low laser power (1-4\%) with the same laser line.

Data processing. Image processing was performed with $\mathrm{NIH}$ ImageJ $(\mathrm{NIH}$, Bethesda, MD, USA). Briefly, stacks of images were deconvolved and rendered into maximum intensity projections for display or average projections for quantification. For quantification purposes, images were thresholded for segmentation. Bleach correction was applied to all time stacks by using a histogram matching macro (Kota Miura and Jens Rietdorf, EMBL Heidelberg, Germany). Quantification of cellular hypertrophy in cardiomyocytes, colocalization analysis as well as particle tracking procedures has been described earlier. ${ }^{24}$ Analysis of mitochondrial morphology was carried out by an ImageJ macro that automates the measurement of the mitochondrial morphological parameters. ${ }^{39,40}$ For FRAP analysis, background corrected images were smoothed with a median filter, and recovery in the bleached region was quantified and corrected for bleaching that occurred during acquisition at low laser power as described. ${ }^{41}$ The recovery (mobile fraction) was calculated as described elsewhere. ${ }^{42}$ All images were assembled in Adobe Illustrator (Adobe Systems, San Jose, CA, USA).

Statistical analysis. Experiments were repeated independently at least three times and archetypal data are presented. Statistical evaluation was carried out using Prism 5 (GraphPad Software, La Jolla, CA, USA). Unless stated otherwise, data represent mean \pm S.E.M. Means were compared by unpaired, two-tailed Student's $t$-test between two groups or one-way analysis of variance, followed by post hoc comparisons with the Tukey's test between multiple groups were performed. $P<0.05$ was considered statistically significant.

\section{Conflict of Interest}

The authors declare no conflict of interest.

Acknowledgements. The study was supported by a grant to $A B$ from the Council of Scientific and Industrial Research (CSIR), Government of India (no. BSC 0206). PB was supported by predoctoral research fellowship from CSIR. We thank Swapan Mondal, Thangamuniyadi Muruganandan and Diptadeep Sarkar for laboratory support and technical assistance. AB gratefully acknowledges the Central Confocal and Atomic Force Microscopy Facility at IICB (funded by CSIR) and CSIRIICB Live Cell Imaging Facility co-funded by the Wellcome Trust and CSIR for the imaging experiments.

\section{Author contributions}

$A B$ and $P B$ designed the study and contributed reagents. $P B$ performed experiments. $P B$ and $A B$ analyzed the data. VC cultured NRVMs and performed assays on NRVMs. $A B$ and $P B$ wrote the manuscript.

1. Hotchkiss RS, Strasser A, McDunn JE, Swanson PE. Cell death. N Engl J Med 2009; 361: 1570-1583.

2. Chiong M, Wang ZV, Pedrozo Z, Cao DJ, Troncoso R, Ibacache M et al. Cardiomyocyte death: mechanisms and translational implications. Cell Death Dis 2011; 2: e244.

3. van Empel VP, De Windt LJ. Myocyte hypertrophy and apoptosis: a balancing act. Cardiovasc Res 2004; 63: 487-499.

4. Gill C, Mestril R, Samali A. Losing heart: the role of apoptosis in heart disease - a novel therapeutic target? FASEB J 2002; 16: 135-146.

5. Frey N, Olson EN. Cardiac hypertrophy: the good, the bad, and the ugly. Annu Rev Physiol 2003; 65: 45-79.

6. Hunter JJ, Chien KR. Signaling pathways for cardiac hypertrophy and failure. N Engl J Med 1999; 341: 1276-1283.

7. van Empel VP, Bertrand AT, Hofstra L, Crijns HJ, Doevendans PA, De Windt LJ. Myocyte apoptosis in heart failure. Cardiovasc Res 2005; 67: 21-29.

8. Hill JA, Olson EN. Cardiac plasticity. N Engl J Med 2008; 358: 1370-1380.

9. Monastyrskaya K, Babiychuk EB, Draeger A. The annexins: spatial and temporal coordination of signaling events during cellular stress. Cell Mol Life Sci 2009; 66: 2623-2642. 10. Moss SE, Morgan RO. The annexins. Genome Biol 2004; 5: 219.
11. Camors E, Monceau V, Charlemagne D. Annexins and $\mathrm{Ca}^{2+}$ handling in the heart. Cardiovasc Res 2005; 65: 793-802.

12. Enrich C, Rentero C, de Muga SV, Reverter M, Mulay V, Wood P et al. Annexin A6-linking $\mathrm{Ca}(2+)$ signaling with cholesterol transport. Biochim Biophys Acta 2011; 1813: 935-947.

13. Vila de Muga S, Timpson P, Cubells L, Evans R, Hayes TE, Rentero $C$ et al. Annexin A6 inhibits Ras signalling in breast cancer cells. Oncogene 2009; 28: 363-377.

14. Podszywalow-Bartnicka P, Kosiorek M, Piwocka K, Sikora E, Zablocki K, Pikula S. Role of annexin $\mathrm{A} 6$ isoforms in catecholamine secretion by $\mathrm{PC} 12$ cells: distinct influence on calcium response. J Cell Biochem 2010; 111: 168-178.

15. Koese M, Rentero C, Kota BP, Hoque M, Cairns R, Wood P et al. Annexin A6 is a scaffold for PKCalpha to promote EGFR inactivation. Oncogene 2012; 32 : 2858-2872.

16. Hawkins TE, Roes J, Rees D, Monkhouse J, Moss SE. Immunological development and cardiovascular function are normal in annexin VI null mutant mice. Mol Cell Biol 1999; 19: 8028-8032.

17. Chlystun M, Campanella M, Law AL, Duchen MR, Fatimathas L, Levine TP et al. Regulation of mitochondrial morphogenesis by annexin a6. PLoS One 2013; 8: e53774.

18. Raynal P, Pollard HB. Annexins: the problem of assessing the biological role for a gene family of multifunctional calcium- and phospholipid-binding proteins. Biochim Biophys Acta 1994; 1197: 63-93.

19. Gunteski-Hamblin AM, Song G, Walsh RA, Frenzke M, Boivin GP, Dorn GW II et al. Annexin $\mathrm{VI}$ overexpression targeted to heart alters cardiomyocyte function in transgenic mice. Am J Physiol 1996; 270: H1091-H1100.

20. Song G, Harding SE, Duchen MR, Tunwell R, O'Gara P, Hawkins TE et al. Altered mechanical properties and intracellular calcium signaling in cardiomyocytes from annexin 6 null-mutant mice. FASEB J 2002; 16: 622-624.

21. Song G, Campos B, Wagoner LE, Dedman JR, Walsh RA. Altered cardiac annexin mRNA and protein levels in the left ventricle of patients with end-stage heart failure. $J$ Mol Cell Cardiol 1998; 30: 443-451.

22. Trouve P, Legot S, Belikova I, Marotte F, Benevolensky D, Russo-Marie F et al. Localization and quantitation of cardiac annexins II, V, and VI in hypertensive guinea pigs. Am J Physiol 1999; 276: H1159-H1166.

23. Mishra S, Chander V, Banerjee P, Oh JG, Lifirsu E, Park WJ et al. Interaction of annexin A6 with alpha actinin in cardiomyocytes. BMC Cell Biol 2011; $12: 7$.

24. Banerjee P, Bandyopadhyay A. Cytosolic dynamics of annexin A6 trigger feedback regulation of hypertrophy via atrial natriuretic peptide in cardiomyocytes. J Biol Chem 2014; 289: 5371-5385.

25. Watkins SJ, Borthwick GM, Arthur HM. The H9C2 cell line and primary neonatal cardiomyocyte cells show similar hypertrophic responses in vitro. In vitro cellular \& developmental biology. Animal 2011; 47: 125-131.

26. Alkistis Frentzou G, Collier ME, Seymour AM, Ettelaie C. Differential induction of cellular proliferation, hypertrophy and apoptosis in $\mathrm{H} 9 \mathrm{c} 2$ cardiomyocytes by exogenous tissue factor. Mol Cell Biochem 2010; 345: 119-130.

27. Mann DL, Bristow MR. Mechanisms and models in heart failure: the biomechanical model and beyond. Circulation 2005; 111: 2837-2849.

28. Maity S, Kar D, De K, Chander V, Bandyopadhyay A. Hyperthyroidism causes cardiac dysfunction by mitochondrial impairment and energy depletion. J Endocrinol 2013; 217: 215-228.

29. Archer SL. Mitochondrial dynamics - mitochondrial fission and fusion in human diseases. N Engl J Med 2013; 369: 2236-2251.

30. Hoppins S, Nunnari J. Cell Biology. Mitochondrial dynamics and apoptosis - the ER connection. Science 2012; 337: 1052-1054.

31. Nagendran J, Gurtu V, Fu DZ, Dyck JR, Haromy A, Ross DB et al. A dynamic and chamberspecific mitochondrial remodeling in right ventricular hypertrophy can be therapeutically targeted. J Thorac Cardiovasc Surg 2008; 136: 168-178.

32. Hazarika P, Kaetzel MA, Sheldon A, Karin NJ, Fleischer S, Nelson TE. Annexin VI is associated with calcium-sequestering organelles. J Cell Biochem 1991; 46: 78-85.

33. Rainteau $D$, Mansuelle $P$, Rochat $H$, Weinman $S$. Characterization and ultrastructural localization of annexin VI from mitochondria. FEBS Lett 1995; 360: 80-84.

34. Putinski C, Abdul-Ghani M, Stiles R, Brunette S, Dick SA, Fernando P. Intrinsic-mediated caspase activation is essential for cardiomyocyte hypertrophy. Proc Natl Acad Sci USA 2013; 110: E4079-E4087.

35. van Berlo JH, Maillet M, Molkentin JD. Signaling effectors underlying pathologic growth and remodeling of the heart. J Clin Invest 2013; 123: 37-45.

36. Karbowski M, Youle RJ. Dynamics of mitochondrial morphology in healthy cells and during apoptosis. Cell Death Differ 2003; 10: 870-880.

37. D'Amours D, Sallmann FR, Dixit VM, Poirier GG. Gain-of-function of poly(ADP-ribose) polymerase-1 upon cleavage by apoptotic proteases: implications for apoptosis. J Cell Sci 2001; 114: 3771-3778.

38. Xiao CY, Chen M, Zsengeller Z, Li H, Kiss L, Kollai M et al. Poly(ADP-Ribose) polymerase promotes cardiac remodeling, contractile failure, and translocation of apoptosis-inducing factor in a murine experimental model of aortic banding and heart failure. J Pharmacol Exp Ther 2005; 312: 891-898.

39. Geistrikh I, Visochek L, Klein R, Miller L, Mittelman L, Shainberg A et al. $\mathrm{Ca}^{2+}$-induced PARP-1 activation and ANF expression are coupled events in cardiomyocytes. Biochem $J$ 2011; 438: 337-347. 
40. Chaitanya GV, Steven AJ, Babu PP. PARP-1 cleavage fragments: signatures of cell-death proteases in neurodegeneration. Cell Commun Signal 2010; 8: 31.

41. Soldani C, Lazze MC, Bottone MG, Tognon G, Biggiogera M, Pellicciari CE et al Poly(ADP-ribose) polymerase cleavage during apoptosis: when and where?. Exp Cell Res 2001; 269: 193-201.

42. Creutz CE, Snyder SL. Interactions of annexins with the mu subunits of the clathrin assembly proteins. Biochemistry 2005; 44: 13795-13806.

43. Jin Z, El-Deiry WS. Overview of cell death signaling pathways. Cancer Biol Ther 2005; 4 $139-163$

44. Guo X, Wang XF. Signaling cross-talk between TGF-beta/BMP and other pathways. Cell Res 2009; 19: 71-88.

45. Robin Donaldson MC. Modular modelling of signalling pathways and their cross- talk. Theor Comput Sci 2012; 456: 30-50

46. Ishihara N, Jofuku A, Eura Y, Mihara K. Regulation of mitochondrial morphology by membrane potential, and DRP1-dependent division and FZO1-dependent fusion reaction in mammalian cells. Biochem Biophys Res Commun 2003; 301: 891-898.

47. Legros F, Lombes A, Frachon P, Rojo M. Mitochondrial fusion in human cells is efficient, requires the inner membrane potential, and is mediated by mitofusins. Mol Biol Cell 2002; 13: 4343-4354.

48. Mattenberger $Y$, James $\mathrm{DI}$, Martinou JC. Fusion of mitochondria in mammalian cells is dependent on the mitochondrial inner membrane potential and independent of microtubules or actin. FEBS Lett 2003; 538: 53-59.

49. Rambold AS, Kostelecky B, Elia N, Lippincott-Schwartz J. Tubular network formation protects mitochondria from autophagosomal degradation during nutrient starvation. Proc Natl Acad Sci USA 2011; 108: 10190-10195.

50. Dorn GW II. Mitochondrial dynamics in heart disease. Biochim Biophys Acta 2013; 1833 233-241.

51. Singh K, Xiao L, Remondino A, Sawyer DB, Colucci WS. Adrenergic regulation of cardiac myocyte apoptosis. J Cell Physiol 2001; 189: 257-265.

52. Xu L, Deng Y, Feng L, Li D, Chen X, Ma C et al. Cardio-protection of salvianolic acid B through inhibition of apoptosis network. PLoS One 2011; 6: e24036.

53. Adams JW, Sakata Y, Davis MG, Sah VP, Wang Y, Liggett SB et al. Enhanced Galphaq signaling: a common pathway mediates cardiac hypertrophy and apoptotic heart failure. Proc Natl Acad Sci USA 1998; 95: 10140-10145.

54. Heineke J, Molkentin JD. Regulation of cardiac hypertrophy by intracellular signalling pathways. Nat Rev Mol Cell Biol 2006; 7: 589-600.
55. Choudhury S, Bae S, Kumar SR, Ke Q, Yalamarti B, Choi JH. Role of AlF in cardiac apoptosis in hypertrophic cardiomyocytes from Dahl salt-sensitive rats. Cardiovasc Res 2010; 85: 28-37.

56. Kuzmicic J, Del Campo A, Lopez-Crisosto C, Morales PE, Pennanen C, Bravo-Sagua R et al. Mitochondrial dynamics: a potential new therapeutic target for heart failure. Rev Esp Cardiol 2011; 64: 916-923

57. Pei Y, Tuschl T. On the art of identifying effective and specific siRNAs. Nat Methods 2006; 3 : 670-676.

58. Eltze $T$, Boer $R$, Wagner $T$, Weinbrenner S, McDonald MC, Thiemermann $C$ et al Imidazoquinolinone, imidazopyridine, and isoquinolindione derivatives as novel and potent inhibitors of the poly(ADP-ribose) polymerase (PARP): a comparison with standard PARP inhibitors. Mol Pharmacol 2008; 74: 1587-1598.

59. Gomez JP, Potreau D, Raymond G. Intracellular calcium transients from newborn rat cardiomyocytes in primary culture. Cell Calcium 1994; 15: 265-275.

60. Bandyopadhyay A, Shin DW, Ahn JO, Kim DH. Calcineurin regulates ryanodine receptor/ $\mathrm{Ca}^{2+}$-release channels in rat heart. Biochem $\mathrm{J} 2000$; 352: 61-70.

61. De K, Ghosh G, Datta M, Konar A, Bandyopadhyay J, Bandyopadhyay D et al. Analysis of differentially expressed genes in hyperthyroid-induced hypertrophied heart by cDNA microarray. J Endocrinol 2004; 182: 303-314.

62. Schmittgen TD, Livak KJ. Analyzing real-time PCR data by the comparative $C(T)$ method. Nat Protoc 2008; 3: 1101-1108.

63. Mitra K, Lippincott-Schwartz J. Analysis of mitochondrial dynamics and functions using imaging approaches. Curr Protocol Cell Biol 2010; Chapter 44: unit 4.25.1-21. published by Nature Publishing Group. This work is licensed under a Creative Commons Attribution 4.0 International License. The images or other third party material in this article are included in the article's Creative Commons license, unless indicated otherwise in the credit line; if the material is not included under the Creative Commons license, users will need to obtain permission from the license holder to reproduce the material. To view a copy of this license, visit http://creativecommons.org/licenses/by/4.0/

Supplementary Information accompanies this paper on Cell Death and Disease website (http://www.nature.com/cddis) 